\title{
Generating set search using simplex gradients for bound-constrained black-box optimization
}

\author{
Sander Dedoncker • Wim Desmet • Frank Naets
}

the date of receipt and acceptance should be inserted later

\begin{abstract}
The optimization problems arising in modern engineering practice are increasingly simulation-based, characterized by extreme types of nonsmoothness, the inaccessibility of derivatives, and high computational expense. While generating set searches (GSS) generally offer a satisfying level of robustness and converge to stationary points, the convergence rates may be slow. In order to accelerate the solution process without sacrificing robustness, we introduce (simplex) gradient-informed generating set search (GIGS) methods for solving bound-constrained minimization problems. These algorithms use simplex gradients, acquired over several iterations, as guidance for adapting the search stencil to the local topography of the objective function. GIGS is shown to inherit first-order convergence properties of GSS and to possess a natural tendency for avoiding saddle points. Numerical experiments are performed on an academic set of smooth, nonsmooth and noisy test problems, as well as a realistic engineering case study. The results demonstrate that including simplex gradient information enables computational cost savings over non-adaptive GSS methods.
\end{abstract}

Keywords derivative-free optimization - bound-constrained optimization · generating set search $\cdot$ simplex derivatives

\section{Introduction}

In recent years, it has become clear that derivative-free optimization (DFO) was and continues to be an indispensable tool in many quantitative disciplines. While gradient-based methods perform very well in theory, the current reality is that many practical optimization problems simply do not satisfy the assumptions of differentiability and transparency that are necessary for their application. As highly complex software environments have become ubiquitous, the objective functions and constraints appearing in relevant problems are often difficult or impossible to analyze in detail and can only be considered as black-boxes. Furthermore, these functional relationships

Correspondence: Sander Dedoncker

KU Leuven, Department of Mechanical Engineering

Celestijnenlaan 300 box 2420, B-3001 Leuven, Belgium

E-mail: sander.dedoncker@kuleuven.be

Sander Dedoncker [0] - Wim Desmet · Frank Naets

KU Leuven, Department of Mechanical Engineering

DMMS lab, Flanders Make 
may suffer from severe (numerical) noise and may not always produce meaningful values (e.g. code errors). The practical need for procedures that can handle black-box optimization (BBO) and, in particular, simulation-based optimization (SBO) is certainly high. The apparent revival of academic research in DFO, BBO and SBO (as surveyed in [13, 8, 29]) is a testament to this.

The subject of the present paper is to develop an original class of algorithms for solving constrained optimization problems of the very general form

$$
\min _{\mathbf{x} \in \Omega} f(\mathbf{x}),
$$

where $f$ is the objective function depending on the design vector $\mathbf{x} \in \mathbb{R}^{n}$, and $\Omega \subset \mathbb{R}^{n}$ represents the feasible set. We focus in particular on the bound-constrained case, so

$$
\Omega=\left\{\mathbf{x} \mid \mathbf{b}_{l} \leq \mathbf{x} \leq \mathbf{b}_{u}\right\} .
$$

Here, $\mathbf{b}_{l}$ and $\mathbf{b}_{u}$ are vectors providing element-wise lower and upper bounds on the design vector. Having in mind SBO and BBO in engineering, the bound-constrained formulation (1) - (2) already covers many interesting applications. In practice, truly unconstrained cases are quite rare, simply because the design variables mostly represent physical quantities. More complex design restrictions do occur, but these are often difficult to express as an explicit constraint function. Therefore, such constraints are assumed to be implicitly encoded in an extended-valued function $f: \mathbb{R}^{n} \rightarrow \mathbb{R} \cup\{+\infty\}$.

Unlike most traditional gradient-based approaches, methods of the directional direct search type can be successfully applied in the SBO context, $[5$, 8. These methods are understood as derivative-free, feasible-point optimization algorithms that proceed by, in each iteration, taking several trial steps from a current iterate and looking for relative objective improvements. In directional direct search, the detrimental effects of high-frequency, low-amplitude noise are mitigated by the inherent, discrete sampling procedure 27. The intrinsically high level of algorithmic robustness allows the treatment of black-box objective functions mapping a heterogeneous set of input parameters to noisy, nonsmooth and/or extended output values.

The use of directional direct search is almost as old as computerized optimization itself [18]. Hooke and Jeeves 24] first introduced the descriptive term pattern search. Over the years, the ideas have been refined and generalized several times [36, 37. In their sizable review paper, Kolda et al. 27] coined the term generating set search (GSS) to denote a group of algorithms including the above examples. Later a somewhat different generalization to pattern search, dubbed mesh adaptive direct search (MADS), was introduced [7. The works [27, 13, 5, 8, 29] give good overviews on directional direct search (GSS and MADS) and related methods.

In the present work, we propose a GSS method that preserves the aforementioned robustness while exploiting the information acquired from earlier iterations. This is motivated by the intuition that it is wasteful to completely disregard this information, even if practical optimization problems commonly feature badly behaved functions. Our method takes the previous evaluations into account by forming linear models and accumulating the associated simplex gradients over the iterations. The resulting global sensitivity indicator is used to reorient the search pattern towards directions of higher sensitivity. We call this method (simplex) gradient-informed generating set search, or GIGS for short.

While the use of simplex gradients for directly determining the search pattern is novel to the best of our knowledge, previous works have explored applications of the concept in the DFO context. Kelley et al. employ purposefully coarse finite-difference gradient approximations - a special case of the simplex gradient - in the implicit filtering method [21, 10, 26. Next, as the name already tends to suggest, simplicial searches such as the Nelder-Mead method naturally accommodate simplex gradients. For instance, in [25, they are used to detect stagnation and 
restart the search, while Tseng 38 proposes their application as a convergence measure. Because simplex gradients are closely related to linear interpolation and regression, the concept also features prominently in model-based DFO approaches [8, 13. Custódio et al. 16, 14, 15, highlight the potential of using simplex derivatives (gradients and Hessians) for poll ordering in generalized pattern searches. Finally, the curvature-informed GSS method from [19, 4] bears a resemblance to our approach, but instead aims to align the search directions with the eigenvectors of an approximate Hessian.

The remainder of the paper is structured as follows: in Section 2, the generic structure of the GSS method is recalled. An introduction to simplex gradients follows in Section 3. Having treated the fundamental concepts and terminology, the subsequent Section 4 presents the new algorithms. The convergence analysis is discussed in Sections 5 and 6. We gather the results of our numerical experiments in Section 7. Section 8 concludes the text.

\section{Generating set search}

As a primer, we give here the outlines of a basic GSS method, as originally defined in [27]. A GSS algorithm starts from a given (feasible) starting point $\mathbf{x}_{0} \in \Omega$, and then proceeds iteratively towards a stationary point $\mathbf{x}_{*}$. This is done by sampling the objective function at certain points and comparing the function values between the samples. Other than population-based methods such as particle swarm [17] or genetic algorithms [23, the sample points are chosen more or less locally and in a structured manner: they form a specific pattern or stencil. The size and shape of the stencil are given by a scalar step length control parameter $\delta_{k}>0$ and by a set of search directions

$$
\mathcal{S}_{k}=\left\{\mathbf{s}_{k}^{(1)}, \mathbf{s}_{k}^{(2)}, \ldots, \mathbf{s}_{k}^{\left(m_{k}\right)}\right\} \subset \mathbb{R}^{n},
$$

where $k \in \mathbb{N}$ denotes the number of the current iteration, and $m_{k}=\left|\mathcal{S}_{k}\right|$.

It is immediately clear that the choice of these vectors $\mathbf{s}_{k}^{(i)}$ is key to the performance of the algorithm. In fact, it determines not only the progress rate, but also whether convergence can be guaranteed. The latter is only possible if the set $\mathcal{S}_{k}$ is rich enough to detect any direction of feasible descent. In unconstrained optimization, for instance, the search directions should include a positive basis for $\mathbb{R}^{n}$. When the problem is constrained, the directions have to adapt to the local geometry of the feasible set. We adopt the following methodology in order to meet this requirement. The search directions $\mathcal{S}_{k}$ are first constructed assuming the absence of constraints, and then modified to conform to the nearby boundary (indicated by a tilde):

$$
\mathcal{S}_{k}=\left\{\mathbf{s}_{k}^{(i)}\right\} \rightarrow \tilde{\mathcal{S}}_{k}=\left\{\tilde{\mathbf{s}}_{k}^{(i)}\right\}
$$

In addition, the individual step lengths $\delta_{k}^{(i)}$ may be shrunk to prevent any steps across the boundary of $\Omega$ :

$$
\delta_{k}^{(i)}=\delta_{k} \rightarrow \tilde{\delta}_{k}^{(i)} \in\left[0 ; \delta_{k}\right]
$$

Following these updates, the trial points $\mathbf{x}_{k}^{(i)}$ in the stencil become

$$
\mathbf{x}_{k}^{(i)}=\mathbf{x}_{k}+\tilde{\delta}_{k}^{(i)} \tilde{\mathbf{s}}_{k}^{(i)}, \quad i \in\left\{1,2, \ldots, m_{k}\right\}
$$

In every iteration, the objective value $f_{k}$ at the current iterate $\mathbf{x}_{k}$ is compared to the values $f_{k}^{(i)}$ at (some of) the trial points. For selecting the next iterate, the realized objective drop is 
measured against a threshold value determined by a forcing function $\rho: \mathbb{R} \rightarrow \mathbb{R}^{+}$that ensures sufficient decrease. If we have

$$
f_{k}-f_{k}^{(i)} \geq \rho\left(\delta_{k}\right)
$$

for one of the trial points $\mathbf{x}_{k}^{(i)}$, then this point can be chosen as the new iterate $\mathbf{x}_{k+1}$. In case this happens, the iteration is called successful. If, on the other hand, no trial points are found satisfying the test (7), the iteration is deemed unsuccessful.

The above process is called polling and has two variants in general. The first is complete polling, where the full stencil is sampled and $\mathbf{x}_{k+1}$ is selected as the best among all candidates (including $\mathbf{x}_{k}$ ). In an effort to reduce the number of function evaluations, opportunistic polling can be introduced. Opportunistic polling assumes that the set of search directions has been assigned a particular order. The trial points are then sampled in the given order and the first point to give an acceptable decrease is taken to be the next iterate. If no such point is found, $\mathbf{x}_{k}$ becomes the next iterate.

Based on the result of the poll, the stencil size is changed by modifying the step length control parameter. Its initial value is $\delta_{0}$, a strictly positive number. As the algorithm progresses, successful iterations are followed by an upscaling of the parameter with the expansion factor $\phi_{k} \geq 1:$

$$
\delta_{k+1}=\phi_{k} \delta_{k} .
$$

Note that $\phi_{k}=1$, i.e. no expansion, is also a possibility. Upon unsuccessful iterations, the step length is downscaled with the contraction factor $\theta_{k}$

$$
\delta_{k+1}=\theta_{k} \delta_{k} .
$$

Here $0<\theta_{k}<\theta_{\max }<1$ (contraction is a strict requirement). The step length control parameter is used to define the main convergence criterion: the algorithm will typically iterate until the step length control parameter drops below some user-defined threshold $\delta_{t o l}$. Of course, other budget constraints such as limits on the number of function evaluations or elapsed time can also be considered to bring about an earlier termination.

\section{Simplex gradients}

A simplex gradient $\nabla_{s} f(\mathbf{x})$ is an approximation of a function gradient $\nabla f(\mathbf{x})$ at a central point $\mathbf{x}$, obtained by constructing a linear model on a set of points surrounding $\mathbf{x}$, as follows. Suppose that $f$ is sampled at $m+1$ points $\mathbf{x}^{(i)}$,

$$
f^{(i)}=f\left(\mathbf{x}^{(i)}\right), \quad i \in\{0,1,2, \ldots, m\}
$$

where $\mathbf{x}^{(0)}$ represents the central point in the sample set. For this set, define the approximate simplex diameter as $\delta_{\max }=\max _{i}\left\|\mathbf{x}^{(i)}-\mathbf{x}^{(0)}\right\|$. To facilitate notation, a set of $m$ vectors in $\mathbb{R}^{n}$ may henceforth be considered as a matrix in $\mathbb{R}^{n \times m}$. This way, define the matrix $\Delta \mathbf{X} \in \mathbb{R}^{n \times m}$ and the vector $\Delta \mathbf{F} \in \mathbb{R}^{m}$ as

$$
\begin{aligned}
& \Delta \mathbf{X}=\left[\mathbf{x}^{(1)}-\mathbf{x}^{(0)}, \mathbf{x}^{(2)}-\mathbf{x}^{(0)}, \ldots, \mathbf{x}^{(m)}-\mathbf{x}^{(0)}\right], \\
& \Delta \mathbf{F}=\left[f^{(1)}-f^{(0)}, f^{(2)}-f^{(0)}, \ldots, f^{(m)}-f^{(0)}\right]^{T} .
\end{aligned}
$$


The simplex gradient $\nabla_{s} f\left(\mathbf{x}^{(0)}\right)$ is then found as the solution to the (least-squares) system

$$
\nabla_{s} f\left(x^{(0)}\right) \in \arg \min _{\mathbf{w}}\left\|\Delta \mathbf{X}^{T} \mathbf{w}-\boldsymbol{\Delta} \mathbf{F}\right\|^{2} .
$$

In case $\operatorname{rank}(\boldsymbol{\Delta} \mathbf{X})=n$, there is a unique solution given by the normal equation

$$
\nabla_{s} f\left(\mathbf{x}^{(0)}\right)=\left(\boldsymbol{\Delta} \mathbf{X} \boldsymbol{\Delta} \mathbf{X}^{T}\right)^{-1} \boldsymbol{\Delta} \mathbf{X} \boldsymbol{\Delta} \mathbf{F} .
$$

Otherwise, the system is underdetermined. We then define $\nabla_{s} f\left(\mathbf{x}^{(0)}\right)$ to be the minimum-norm solution to (13). This approach desensitizes the gradient estimate to perturbations on $\boldsymbol{\Delta} \mathbf{X}$ and, more importantly, prevents the intrusion of vector components orthogonal to the columns of $\Delta \mathbf{X}$ [12]. The Moore-Penrose pseudo-inverse is used to concisely represent both cases as

$$
\nabla_{s} f\left(\mathbf{x}^{(0)}\right)=\left(\Delta \mathbf{X}^{T}\right)^{\dagger} \boldsymbol{\Delta} \mathbf{F} .
$$

To ensure that the simplex gradient is an accurate approximation to the real gradient, the geometry of the sample set is an important factor. This is summarized in the following theorem, which can be found in e.g. [12, 16]

Theorem 1 (Error bound on the gradient approximation). Let $\left\{\mathbf{x}^{(0)}, \mathbf{x}^{(1)}, \ldots, \mathbf{x}^{(m)}\right\} \subset \mathbb{R}^{n}$ be a sample set for constructing the simplex gradient $\nabla_{s} f\left(\mathbf{x}^{(0)}\right)$. Assume that $\operatorname{rank}(\boldsymbol{\Delta} \mathbf{X})=$ $\min (m, n)$. Let $\hat{\mathbf{U}}_{X} \hat{\boldsymbol{\Sigma}}_{X} \hat{\mathbf{V}}_{X}^{T}$ represent the reduced ('economy-size') singular value decomposition of $\boldsymbol{\Delta} \mathbf{X}^{T} / \delta_{\max }$. Assume that $\nabla f$ is Lipschitz continuous on an open domain containing the enclosing ball $\mathcal{B}\left(\mathbf{x}^{(0)} ; \delta_{\max }\right)$ with constant $\gamma>0$. The error of the simplex gradient at $\mathbf{x}^{(0)}$, as an approximation to $\nabla f\left(\mathbf{x}^{(0)}\right)$, then satisfies

$$
\left\|\hat{\mathbf{V}}_{X}^{T}\left[\nabla_{s} f\left(\mathbf{x}^{(0)}\right)-\nabla f\left(\mathbf{x}^{(0)}\right)\right]\right\| \leq\left(\sqrt{m} \frac{\gamma}{2}\left\|\hat{\boldsymbol{\Sigma}}_{X}^{-1}\right\|\right) \delta_{\max } .
$$

Note that the error can only be bounded as far as the component inside the column space of $\hat{\mathbf{V}}_{X}$ (which is that of $\boldsymbol{\Delta} \mathbf{X}$ ) is concerned, which is reasonable as the sensitivity of $f$ in other directions is not revealed using this sample set. If there are at least $n$ linearly independent columns in $\Delta \mathbf{X}$, then $\hat{\mathbf{V}}_{X}$ is square and $(15)$ is a total error bound. The theorem shows that the simplex gradient error is linear in the approximate simplex diameter $\delta_{\max }$ and that the constant depends on the sample set geometry through $\left\|\hat{\boldsymbol{\Sigma}}_{X}^{-1}\right\|$. Well-poised sample sets minimize this quantity by using steps of similar lengths in dissimilar directions.

\section{Adaptive GSS using simplex gradients}

\subsection{Basic approach}

Conceptually, the calculation of simplex gradients and the sampling strategy in GSS methods clearly have a lot in common. In every iteration of GSS, it is possible to construct a simplex gradient of the objective function at the current iterate. Since the necessary function evaluations are already taken care of by the GSS algorithm, the added cost of this procedure is minimal - an important consideration if the objective is expensive to evaluate. Of course, the simplex gradient is only known as soon as the samples have been taken; it is always effectively a step behind the optimization algorithm. Moreover, the gradient is only a single direction, limiting its use for guiding the directional search. To overcome these limitations, we propose to utilize the gradient information accumulated over the iteration history. This information can be processed to a usable form by applying the singular value decomposition (SVD), as shown next. 
Suppose some previously calculated simplex gradients $\mathbf{g}$ are arranged as columns of the matrix $\mathbf{G} \in \mathbb{R}^{n \times q}$, and decompose $\mathbf{G}$ as

$$
\mathbf{G}=\mathbf{U} \boldsymbol{\Sigma} \mathbf{V}^{T},
$$

where $\mathbf{U} \in \mathbb{R}^{n \times n}$ and $\mathbf{V} \in \mathbb{R}^{q \times q}$ are orthogonal, and $\boldsymbol{\Sigma} \in \mathbb{R}^{n \times q}$ is diagonal. We now introduce the left singular vectors $\mathbf{u}^{(i)}$ as search directions in a GSS method. This decision is best explained using the statistical interpretation of the SVD, which views the left singular vectors as the principal directions of variance of the vector samples $\mathbf{g}$. Their components along $\mathbf{u}^{(i)}$ are uncorrelated and ordered by decreasing variance. In this sense, the singular vectors give the directions in which the objective is expected to change the fastest.

\subsection{Generic implementation}

The goal in the remainder of this section is to first define a generic method of combining GSS with simplex gradients and then classify the concrete instances that are further studied. Algorithm 1 describes the common approach, which follows the outline of Section 2 To allow for the variations and to make its description more readable, this main algorithm makes use of the auxiliary procedures from Algorithms 2 and 3 .

As input, the user provides: the objective function $f$, the initial iterate $\mathbf{x}_{0}$, the lower and upper bound vectors $\mathbf{b}_{l}$ and $\mathbf{b}_{u}$, the initial step length control parameter $\delta_{0}$, the step length tolerance $\delta_{\text {tol }}$, the relative constraint activation threshold $\epsilon_{\text {tol }}>0$, the contraction and expansion parameters $\theta$ and $\phi$, the forcing function $\rho$, the number of saved gradient samples $q$, and the number of preserved singular directions $r$. Throughout the optimization process, the search directions, step lengths and poll order are automatically determined from these parameters.

All search directions are collectively stored in the matrix $\mathbf{S}$. We adopt the usual distinction between supplementary search directions $\mathbf{H}$ and core directions $[\mathbf{D}, \mathbf{C}]$. The matrix $\mathbf{D}$ represents an orthogonal positive basis, which we define as follows:

$$
\mathbf{D}=\mathbf{Q}[\mathbf{I},-\mathbf{I}]
$$

where $\mathbf{Q}$ is some orthogonal matrix. The columns of $\mathbf{C}$ contain positive bases for the feasible set boundary - because the constraints are simple bounds, the positive and negative coordinate directions may be used. The sets $\mathbf{H}$ and $\mathbf{D}$ are constructed from two possible sources: the coordinate directions and the singular directions $\mathbf{U}$ of the simplex gradient samples $\mathbf{G}$. The information for determining and ordering these vectors is accumulated over several iterations, allowing adaptation to the local topography of the objective function.

Since the geometry of the stencil should reflect the geometry of the nearby boundary, the basic search directions are further processed before the poll step. The parameter $\epsilon_{\text {tol }}$ dictates which constraints are close enough to be considered. To avoid that search directions are modified too aggressively, $\epsilon_{\text {tol }}$ should be small enough. The convergence analysis allows it to be arbitrarily close to zero, but finite numerical precision could cause some practical problems if it is too small. As soon as the correct search directions are known, the step lengths are individually maximized within the limits of feasibility [28]. Symbols for search directions and step lengths are augmented with a tilde to indicate that the presence of boundaries has been taken into account.

The poll step has options for both complete and opportunistic polling. As a forcing function, any $\rho$ satisfying Lemma 4 is possible (see Section 6). If the objective represents a quantity with an absolute meaning (for instance a physical performance measure), we suggest using

$$
\rho_{k}(t)=\max \left(1,\left|f_{k}\right|\right) \tau_{\text {tol }} t^{2} .
$$


This function prevents long iteration stretches with relatively marginal improvements, without invalidating the convergence analysis. The tuning parameter $\tau_{\text {tol }}$ can be chosen higher if faster termination is desired. Alternatively, the more conventional choice

$$
\rho_{k}(t)=\tau_{\text {tol }} t^{2}
$$

is a valid possibility. Polling yields a next iterate as well as any valid function evaluations with their associated steps $(\overline{\boldsymbol{\Delta} \mathbf{F}}$ and $\overline{\boldsymbol{\Delta S}})$.

If the poll was successful, the stencil is expanded and the search directions are reordered. The procedure [reorder] currently implements a heuristic referred to as dynamic polling [7, where the directions that were last successful are prioritized. This deterministic strategy is preferred for the comparative studies presented below. Note, however, that it is not fundamental to the GIGS method and that other heuristic or random ordering methods could be employed in practice.

After an unsuccessful poll, the matrix $\mathbf{G}$ is updated using the pseudo-inverse formula (14) for computing a new simplex gradient. Because the failed function evaluations have been discarded, they will not cause the algorithm to break down during this computation. While the quality (poisedness) of the sample set is not monitored explicitly, the presence of a positive orthogonal set $\tilde{\mathbf{D}}$ and/or well-conditioned boundary set $\tilde{\mathbf{C}}$ is assumed to mitigate the worst possible situations [16]. Because updates to $\mathbf{G}$ only occur in case of an unsuccessful poll, a substantial number of samples should be available to use.

The algorithm outline of [singdir] gives no details on the construction of the SVD since this computation is standard in most numerical linear algebra packages. The typical application of GSS is characterized by a moderate design dimension and an expensive objective function: in such cases, the cost of the SVD should be relatively small. Otherwise, a low-rank modification algorithm could be exploited to increase efficiency [11].

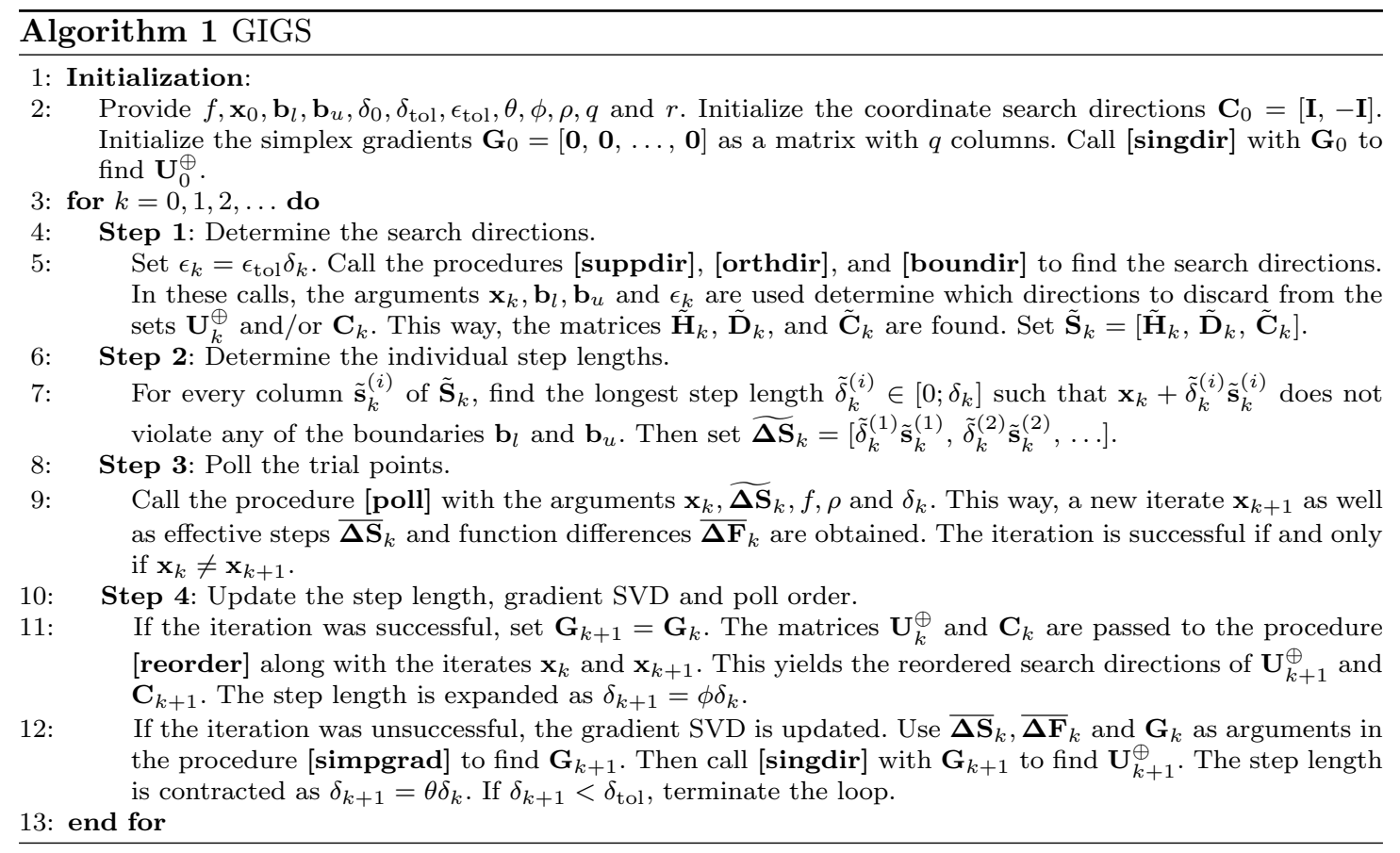




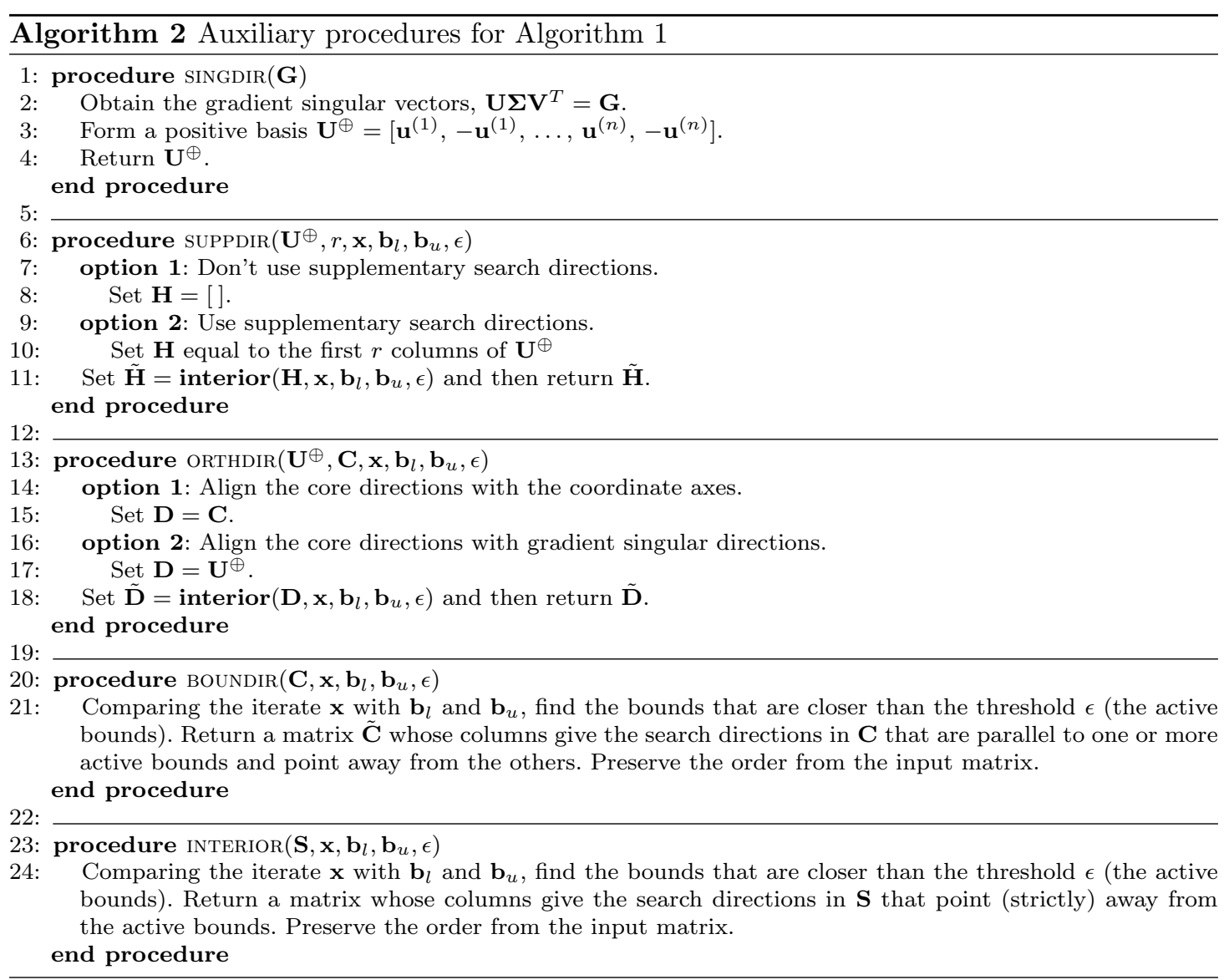

\subsection{Algorithmic variants}

Algorithm 1 has several different versions due to the way [suppdir], [orthdir] and [poll] are defined. Because it is not immediately clear which particular version will be the most practically effective, we report on empirical tests that give an indication about the relative performance in Section 7. To make the presentation less cumbersome, we first provide labels for the algorithmic variants. In the remainder of the text, variant $x$ can then be referred to as 'Algorithm 1 . $x$ '.

The matrix of Table 1 shows the classification. The choices of the orthogonal stencil $\mathbf{D}$, the supplementary stencil $\mathbf{H}$ and the poll method lead to a total of 6 possibilities - since the adaptive choice of $\mathbf{D}$ overlaps with $\mathbf{H}$, the combined option is not included. Variants 1 and 2 represent the conventional coordinate search with complete and opportunistic polling, respectively. Variants 3 to 4 expand on this by including the simplex gradient singular vectors as supplementary search directions. The final two variants, then, align the orthogonal stencil $\mathbf{D}$ with the singular directions.

\section{Convergence analysis}

As a central topic in much of the literature on direct search methods, in particular GSS, associated convergence properties have been extensively analyzed. For smooth functions, strong statements 


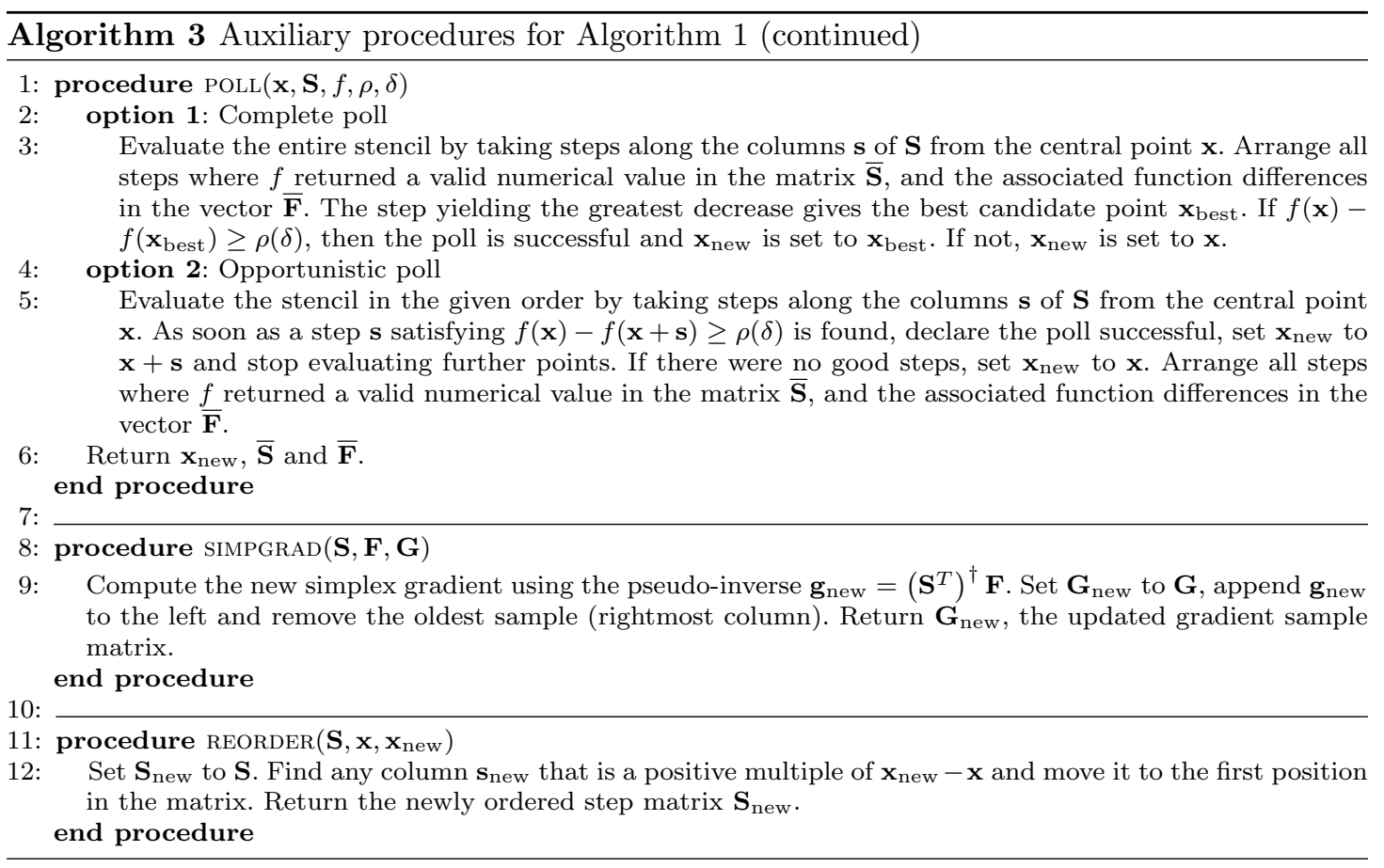

Table 1: Classification of the possible variants of Algorithm 1. Each instance is numbered and given a 3-letter descriptive code. The first letter represents the poll strategy, which can be complete $(\mathrm{C})$ or opportunistic $(\mathrm{O})$. The second letter represents the choice of the orthogonal stencil, which can be aligned with coordinate directions (I) or gradient singular vectors (U). The third letter represents whether supplementary search directions are used (S) or not used (N).

\begin{tabular}{l||c|c||c|} 
& \multicolumn{2}{c}{ D nonadaptive } & D adaptive \\
\hline & H empty & H nonempty & H empty \\
\hline Complete poll & 1 (CIN) & 3 (CIS) & 5 (CUN) \\
Opportunistic poll & 2 (OIN) & 4 (OIS) & 6 (OUN)
\end{tabular}

about asymptotic convergence rates, first-order and second-order stationarity are possible [27, 1, 2, 28. Global convergence can be proved for a much larger class of functions, using concepts from nonsmooth calculus [6, 39. Much of the literature focuses on how these algorithms can be modified to handle constraints of different types. The treatment of linear inequality constraints, in particular bound constraints, was addressed early on by Lewis and Torczon [31, 32, and further studied in [27, 30, 28].

We will make use of the results that were established in the earlier work of Kolda et al. [28] to show GIGS is guaranteed to converge globally to a Karush-Kuhn-Tucker (KKT) point. While the present work emphasizes the bound-constrained case, the following analysis considers the more general class of linearly constrained problems, with feasible set

$$
\Omega=\{\mathbf{x} \mid \mathbf{A x} \leq \mathbf{b}\}
$$

Note that bound-constrained or linear-equality-constrained problems are special cases of the above situation. 
Since a certain freedom in the definition of the search directions is necessary, we rely on the sufficient-decrease strategy for algorithm globalization. Algorithm 5.1 from 28] (henceforth called GSS-SD) provides a template for GSS with sufficient decrease. We proceed by showing that the GIGS variants instantiate GSS-SD.

It is readily verified that all algorithms described in Section 4 fit the general form of the generic GSS-SD algorithm. The salient question is whether or not they comply with the technical conditions that are also part of the definition. As it turns out, this is indeed the case. We state here this important result as a theorem; the detailed analysis and proof is deferred to the next section to enhance the readability.

Theorem 2. Algorithm 1 (GIGS) defines valid instances of GSS-SD.

The convergence properties can hence be directly inferred from [28]. First of all, it is possible to guarantee that the step length control parameter gets arbitrarily small as the algorithms progress. Their termination is certain, since $\delta_{k}>\delta_{t o l}$ is a condition for continuing the iterations.

Theorem 3 ([28, Theorem 5.1). Suppose $f$ is bounded below on $\Omega$. Then GSS-SD has the property

$$
\liminf _{k \rightarrow+\infty} \delta_{k}=0
$$

Note that this result supposes nothing more than the existence of a global minimum on the feasible domain $\Omega$ (as a matter of fact, it holds for nonsmooth $f$ or nonlinear $\Omega$ as well).

The additional assumption that $f$ is smooth enables stronger statements. In the analysis, the quantity $\chi(\mathbf{x})$, measuring stationarity of a point for constrained optimization, plays a central role. It is defined as

$$
\chi(\mathbf{x}) \equiv \max _{\substack{\mathbf{x}+\mathbf{w} \in \Omega \\\|\mathbf{w}\| \leq 1}}-\nabla f(\mathbf{x})^{T} \mathbf{w} \quad \text { for } \quad \mathbf{x} \in \Omega .
$$

and is a continuous, nonnegative function on $\Omega$. Furthermore, $\chi(\mathbf{x})=0$ if and only if $\mathbf{x}$ is a KKT point for the linearly constrained problem of equations (1) and (20). We also define the sublevel set

$$
\mathcal{F}=\left\{\mathbf{x} \in \Omega \mid f(\mathbf{x}) \leq f\left(\mathbf{x}_{0}\right)\right\},
$$

which is a set enveloping all possible iterates $\mathbf{x}_{k}$. Obviously, if $\Omega$ is bounded - which for engineering problems happens more often than not - then so will be $\mathcal{F}$. Making use of these concepts, we can (re)state the next convergence theorem.

Theorem 4 ([28, Theorem 6.4). Suppose that $f$ is continuously differentiable on $\Omega$ with a Lipschitz continuous gradient, and that the set $\mathcal{F}$ is bounded. Then for unsuccessful iterations $k$ in GSS-SD, there exist constants $C_{1}, C_{2}>0$ such that

$$
\chi\left(\mathbf{x}_{k}\right) \leq C_{1} \delta_{k}+C_{2} \frac{\rho\left(\delta_{k}\right)}{\delta_{k}} .
$$

The example functions given by equations $(18)$ and $\sqrt{19}$ yield $\rho\left(\delta_{k}\right) / \delta_{k}=O\left(\delta_{k}\right)$, so we also obtain the useful estimate

$$
\chi\left(\mathbf{x}_{k}\right)=O\left(\delta_{k}\right) .
$$

Theorem 4 provides the mathematical justification for using $\delta_{k}$ as a stopping criterion, since it bounds the stationarity measure $\chi$. As a corollary to Theorems 2-4 the global convergence of the proposed algorithms is demonstrated. 
The above results ensure convergence to a KKT point, but in principle it is still possible that this point is a saddle point or a maximizer. In [22, 4, the second-order convergence of GSS-SD is investigated. A subclass of linearly constrained GSS-SD with orthogonal search directions, based on the curvature-informed GSS algorithm proposed in [19], was shown to find solutions satisfying second-order optimality conditions in the nonsmooth setting [4. Gratton et al. [22] provide second-order stationarity guarantees and worst-case complexity bounds in the unconstrained case. For instances of GSS-SD without orthogonal search directions, they obtain a directional stationarity result. To assure convergence to a point with the strong second-order optimality conditions (positive semidefinite Hessian), both works assume that the limiting search directions are aligned with eigenvectors of the local Hessian.

While we do not use Hessian estimates explicitly, the procedure of aggregating gradients does reveal some information on the curvature of the objective function. Consider the following example. Suppose the function $f(\mathbf{x})$ is locally analytic at the critical point $\mathbf{x}_{*}$. Then, for small enough $\epsilon>0$ and unit vector $\mathbf{t}$ :

$$
f\left(\mathbf{x}_{*}+\epsilon \mathbf{t}\right)=f\left(\mathbf{x}_{*}\right)+\epsilon \nabla f\left(\mathbf{x}_{*}\right)^{T} \mathbf{t}+\frac{\epsilon^{2}}{2} \mathbf{t}^{T} \nabla^{2} f\left(\mathbf{x}_{*}\right) \mathbf{t}+O\left(\epsilon^{3}\right) .
$$

For the remainder of the paragraph we denote the Hessian matrix $\nabla^{2} f\left(\mathbf{x}_{*}\right)$ by $\mathbf{H}$ to make the notation less cumbersome. From the Taylor approximation, we have that

$$
\nabla f\left(\mathbf{x}_{*}+\epsilon \mathbf{t}\right)=\epsilon \mathbf{H t}+O\left(\epsilon^{2}\right)
$$

because $\nabla f\left(\mathbf{x}_{*}\right)=0$. Assume $\mathbf{t}$ to be an isotropically distributed random vector. Under these conditions, the covariance matrix of the gradient becomes

$$
\begin{aligned}
\mathbb{E}\left[\nabla f\left(\mathbf{x}_{*}+\epsilon \mathbf{t}\right) \nabla f\left(\mathbf{x}_{*}+\epsilon \mathbf{t}\right)^{T}\right] & \approx \mathbb{E}\left[\epsilon^{2} \mathbf{H t t}^{T} \mathbf{H}^{T}\right] \\
& =\epsilon^{2} \mathbf{H} \mathbb{E}\left[\mathbf{t t}^{T}\right] \mathbf{H}^{T} \\
& \propto \epsilon^{2} \mathbf{H}^{2} .
\end{aligned}
$$

Since $\mathbf{H}$ is symmetric, it admits an orthogonal eigendecomposition. It follows that

$$
\mathbb{E}\left[\nabla f\left(\mathbf{x}_{*}+\epsilon \mathbf{t}\right) \nabla f\left(\mathbf{x}_{*}+\epsilon \mathbf{t}\right)^{T}\right] \approx \epsilon^{2} \mathbf{U}\left(\boldsymbol{\Sigma} \boldsymbol{\Sigma}^{T}\right) \mathbf{U}^{T},
$$

where $\mathbf{U}$ is a matrix whose columns are the eigenvectors of $\mathbf{H}$ and $\left(\boldsymbol{\Sigma} \boldsymbol{\Sigma}^{T}\right)$ is diagonal. For a sufficiently large number of gradient samples, taken isotropically from a small neighborhood around the minimizer, the eigenvectors of the matrix $\mathbf{G G}^{T}$ (search directions in GIGS) thus approach those of $\mathbf{H}$. It is therefore not unreasonable to expect that the adaptive algorithms can avoid saddle points more easily than, for example, GSS with fixed search directions.

\section{Proof of Theorem 2}

For the sufficient-decrease version of generating set search, 28, presents sufficient conditions for (first-order) convergence. The crux of proving Theorem 2 consists of showing that all variants of Algorithm 1 satisfy a condition similar to [28, Condition 1. This is done in the following part. The other criteria are far easier to verify and will be treated in the second subsection. Preliminary results and definitions (cosine measure, $\epsilon$-active constraints, $\epsilon$-tangent cone) can be found in Appendix A. 
6.1 Condition on cosine measures of the search directions

Loosely speaking, a first requirement is to assure that the feasible descent directions do not get 'arbitrarily orthogonal' to the search directions that are used in the stencil. The following lemma formally states this condition.

Lemma 1 (analogue of [28, Condition 1). For every $k$ with $\mathcal{T}\left(\mathbf{x}_{k}, \epsilon_{k}\right) \neq\{\mathbf{0}\}$, the set $\{\tilde{\mathcal{D}}, \tilde{\mathcal{C}}\}_{k}$ given by the columns of $\left[\tilde{\mathbf{D}}_{k}, \tilde{\mathbf{C}}_{k}\right]$ in Algorithm 1 generates $\mathcal{T}\left(\mathbf{x}_{k}, \epsilon_{k}\right)$ and satisfies $\kappa\left(\{\tilde{\mathcal{D}}, \tilde{\mathcal{C}}\}_{k}\right) \geq$ $\kappa_{\text {min }}$. The constant $\kappa_{\text {min }}>0$ is independent of $k$.

Proof. First of all, let us install some notational simplifications. Since the lemma must hold for any iteration $k$ separately, we drop any reference to this number in the proof. Furthermore, the iterate $\mathbf{x}$ and parameter $\epsilon$ are fixed in any particular iteration, so any explicit dependency on these quantities is omitted. The $\epsilon$-tangent cone $\mathcal{T}\left(\mathbf{x}_{k}, \epsilon_{k}\right)$ and $\epsilon$-active set $\mathcal{I}\left(\mathbf{x}_{k}, \epsilon_{k}\right)$ are hence simply written as $\mathcal{T}$ and $\mathcal{I}$.

Now, briefly consider the sets $\mathcal{D}, \mathcal{C}, \tilde{\mathcal{D}}$ and $\tilde{\mathcal{C}}$ so we can highlight some important structure in them. The set $\mathcal{D}$ is an orthogonal positive basis for $\mathbb{R}^{n}$. Providing this set as input to the procedure [interior] yields $\tilde{\mathcal{D}} \subseteq \mathcal{D}$, in which only those vectors in the interior of the tangent cone $\mathcal{T}$ are preserved. Next, there is $\mathcal{C}$. This set is generally defined to contain within it positive bases for any possible $\partial \mathcal{T}$. In the particular case of simple bound constraints emphasized here, it can for example be made up of all positive and negative coordinate directions. For more general linear constraints, obtaining good-quality generating sets is more difficult, but practical methods for doing so have been described in the literature [30, 3]. The procedure [boundir] isolates from $\mathcal{C}$ those vectors positively spanning $\partial \mathcal{T}$ and returns them as the set $\tilde{\mathcal{C}}$. Note that this set is a function only of $\mathcal{I}$ and that, as a consequence, there are only a finite number of different $\tilde{\mathcal{C}}$.

To prove that the set of core directions $\{\tilde{\mathcal{D}}, \tilde{\mathcal{C}}\}$ generates $\mathcal{T}$ and does so with finite cosine measure, we distinguish three possible situations, and proceed on a case-by-case basis.

$\mathcal{I}=\emptyset$ (no active constraints) If there are no active constraints, then $\mathcal{T}=\mathbb{R}^{n}$ and all vectors are in its interior. This means that $\{\tilde{\mathcal{D}}, \tilde{\mathcal{C}}\}=\mathcal{D}$. Because $\mathcal{D}$ is an orthogonal positive basis for $\mathbb{R}^{n}$, we have 37 .

$$
\kappa_{1}=\kappa(\mathcal{D}) \geq \frac{1}{\sqrt{n}}
$$

$|\mathcal{I}|=1$ (single active constraint) Denote $\mathbf{a}^{T}$ the row of the constraint matrix giving the active constraint. In this case, we are provided with a positive spanning set for $\partial \mathcal{T}$ (which is the kernel of $\mathbf{a}^{T}$ ), namely $\tilde{\mathcal{C}}$. In addition, since $\mathcal{T}$ is a half-space and $\mathcal{D}$ is an orthogonal positive basis for the full space, there is at least one vector of $\mathcal{D}$ in the interior of $\mathcal{T}$. By Proposition 1 (Appendix A), $\{\tilde{\mathcal{D}}, \tilde{\mathcal{C}}\}$ positively generates $\mathcal{T}$. To prove $\kappa(\{\tilde{\mathcal{D}}, \tilde{\mathcal{C}}\})$ can be bounded away from zero, we divide the set with nonzero projection on $\mathcal{T}$ in three disjoint regions:

$$
\begin{aligned}
\left\{\mathbf{w} \mid \mathbf{w}_{\mathcal{T}} \neq 0\right\} & =\mathcal{R}_{a} \cup \mathcal{R}_{b} \cup \mathcal{R}_{c} \text { with } \\
\mathcal{R}_{a} & =\left\{\mathbf{w} \mid 0 \leq \mathbf{w}^{T} \mathbf{a}<\|\mathbf{w}\|\|\mathbf{a}\|, \mathbf{w} \neq \mathbf{0}\right\}, \\
\mathcal{R}_{b} & =\left\{\mathbf{w} \mid-\alpha\|\mathbf{w}\|\|\mathbf{a}\|<\mathbf{w}^{T} \mathbf{a}<0\right\}, \\
\mathcal{R}_{c} & =\left\{\mathbf{w} \mid \mathbf{w}^{T} \mathbf{a} \leq-\alpha\|\mathbf{w}\|\|\mathbf{a}\|, \mathbf{w} \neq \mathbf{0}\right\},
\end{aligned}
$$

where $0<\alpha<1$.

For $\mathbf{w} \in \mathcal{R}_{a}$, we have 


$$
\inf _{\mathbf{w} \in \mathcal{R}_{a}} \max _{\mathbf{d} \in\{\tilde{\mathcal{D}}, \tilde{\mathcal{C}}\}} \frac{\mathbf{w}^{T} \mathbf{d}}{\left\|\mathbf{w}_{\mathcal{T}}\right\|\|\mathbf{d}\|} \geq \inf _{\mathbf{w} \in \mathcal{R}_{a}} \max _{\mathbf{d} \in \tilde{\mathcal{C}}} \frac{\mathbf{w}^{T} \mathbf{d}}{\left\|\mathbf{w}_{\mathcal{T}}\right\|\|\mathbf{d}\|}=\inf _{\mathbf{w} \in \mathcal{R}_{a}} \max _{\mathbf{d} \in \tilde{\mathcal{C}}} \frac{\mathbf{w}^{T} \mathbf{d}}{\left\|\mathbf{w}_{\mathcal{L}(\tilde{\mathcal{C}})}\right\|\|\mathbf{d}\|}=\kappa(\tilde{\mathcal{C}}) .
$$

The first inequality follows from shrinking the set of candidate vectors to $\tilde{\mathcal{C}}$, and the first equality is a consequence of the definition of $\mathcal{R}_{a}$. There are only a finite number of possible $\tilde{\mathcal{C}}$, so we take $\kappa_{2 a}$ to be the minimum of $\kappa(\tilde{\mathcal{C}})$ over this finite set. This minimum is strictly positive.

For $\mathbf{w} \in \mathcal{R}_{b}$, it holds that

$$
\inf _{\mathbf{w} \in \mathcal{R}_{b}} \max _{\mathbf{d} \in\{\tilde{\mathcal{D}}, \tilde{\mathcal{C}}\}} \frac{\mathbf{w}^{T} \mathbf{d}}{\|\mathbf{w} \mathcal{T}\|\|\mathbf{d}\|} \geq \inf _{\mathbf{w} \in \mathcal{R}_{b}} \max _{\mathbf{d} \in \tilde{\mathcal{C}}} \frac{\mathbf{w}^{T} \mathbf{d}}{\|\mathbf{w} \mathcal{T}\|\|\mathbf{d}\|}=\inf _{\mathbf{w} \in \mathcal{R}_{b}} \max _{\mathbf{d} \in \tilde{\mathcal{C}}} \frac{\mathbf{w}^{T} \mathbf{d}}{\|\mathbf{w}\|\|\mathbf{d}\|}>0
$$

The last, strict inequality is a consequence of the fact that within $\mathcal{R}_{b}, \mathbf{w}$ cannot make an arbitrarily small angle with $-\mathbf{a}$. Again, there are finitely many possible $\tilde{\mathcal{C}}$ : we take $\kappa_{2 b}$ to be the minimum of the above quantity over all $\tilde{\mathcal{C}}$.

Considering $\mathbf{w} \in \mathcal{R}_{c}$ requires a little bit more care. Because within this set, w can become orthogonal to all $\mathbf{d} \in \tilde{\mathcal{C}}$, we have

$$
\inf _{\mathbf{w} \in \mathcal{R}_{c}} \max _{\mathbf{d} \in \tilde{\mathcal{C}}} \frac{\mathbf{w}^{T} \mathbf{d}}{\|\mathbf{w} \mathcal{T}\|\|\mathbf{d}\|}=\inf _{\mathbf{w} \in \mathcal{R}_{c}} \max _{\mathbf{d} \in \tilde{\mathcal{C}}} \frac{\mathbf{w}^{T} \mathbf{d}}{\|\mathbf{w}\|\|\mathbf{d}\|}=0 .
$$

As a consequence, the vectors in $\tilde{\mathcal{C}}$ cannot be used in the further argument; we rely on the complementary set $\tilde{\mathcal{D}}$. Recall that by definition, all vectors $\mathbf{d} \in \mathcal{D}$ with $-\mathbf{d}^{T} \mathbf{a}>0$ are preserved in $\tilde{\mathcal{D}}$. Since these vectors are also mutually orthogonal, there exist $\mu_{i}>0$ for which

$$
\begin{aligned}
-\mathbf{a} & =\sum_{\mathbf{d}_{i} \in \tilde{\mathcal{D}}} \mu_{i} \mathbf{d}_{i}, \text { and } \\
\|\mathbf{a}\|^{2} & =\mathbf{a}^{T} \mathbf{a}=\sum_{\mathbf{d}_{i} \in \tilde{\mathcal{D}}} \mu_{i}^{2} \mathbf{d}_{i}^{T} \mathbf{d}_{i} .
\end{aligned}
$$

Now, let $i^{*}$ denote the index that maximizes the quantity $\mu_{i}\left(\mathbf{w}^{T} \mathbf{d}_{i}\right)$. Because of the definition of $\mathcal{R}_{c}$, for all w inside:

$$
\begin{aligned}
0 & <\alpha \leq \frac{-\mathbf{w}^{T} \mathbf{a}}{\|\mathbf{w}\|\|\mathbf{a}\|} \\
& =\frac{\mathbf{w}^{T} \sum_{\mathbf{d}_{i} \in \tilde{\mathcal{D}}} \mu_{i} \mathbf{d}_{i}}{\|\mathbf{w}\| \sqrt{\sum_{\mathbf{d}_{i} \in \tilde{\mathcal{D}}} \mu_{i}^{2} \mathbf{d}_{i}^{T} \mathbf{d}_{i}}}
\end{aligned}
$$

Therefore,

$$
\begin{array}{rll}
\alpha & \leq \frac{\mathbf{w}^{T} \sum_{\mathbf{d}_{i} \in \tilde{\mathcal{D}}} \mu_{i} \mathbf{d}_{i}}{\|\mathbf{w}\| \sqrt{\sum_{\mathbf{d}_{i} \in \tilde{\mathcal{D}}} \mu_{i}^{2} \mathbf{d}_{i}^{T} \mathbf{d}_{i}}} & \\
& \left.\leq \frac{|\tilde{\mathcal{D}}| \mu_{i}^{*}\left(\mathbf{w}^{T} \mathbf{d}_{i}^{*}\right)}{\|\mathbf{w}\| \sqrt{\sum_{\mathbf{d}_{i} \in \tilde{\mathcal{D}}} \mu_{i}^{2} \mathbf{d}_{i}^{T} \mathbf{d}_{i}}} \quad \text { (definition of } i^{*}\right) \\
& \left.\leq \frac{|\tilde{\mathcal{D}}| \mu_{i}^{*}\left(\mathbf{w}^{T} \mathbf{d}_{i}^{*}\right)}{\|\mathbf{w}\| \mu_{i}^{*}\left\|\mathbf{d}_{i}^{*}\right\|}=\frac{|\tilde{\mathcal{D}}| \mathbf{w}^{T} \mathbf{d}_{i}^{*}}{\|\mathbf{w}\|\left\|\mathbf{d}_{i}^{*}\right\|} \quad \text { (square root is monotonic, } \mu_{i}>0\right)
\end{array}
$$

Since there are no more than $n$ orthogonal vectors in a half-space, $|\tilde{\mathcal{D}}| \leq n$ and 


$$
\frac{\alpha}{n} \leq \frac{\mathbf{w}^{T} \mathbf{d}_{i}^{*}}{\|\mathbf{w}\|\left\|\mathbf{d}_{i}^{*}\right\|} \leq \max _{\mathbf{d} \in \tilde{\mathcal{D}}} \frac{\mathbf{w}^{T} \mathbf{d}}{\|\mathbf{w}\|\|\mathbf{d}\|} .
$$

The above statement holds for all $\mathbf{w} \in \mathcal{R}_{c}$, so finally, in analogy to 27) and 28):

$$
\inf _{\mathbf{w} \in \mathcal{R}_{c}} \max _{\mathbf{d} \in\{\tilde{\mathcal{D}}, \tilde{\mathcal{C}}\}} \frac{\mathbf{w}^{T} \mathbf{d}}{\left\|\mathbf{w}_{\mathcal{T}}\right\|\|\mathbf{d}\|} \geq \inf _{\mathbf{w} \in \mathcal{R}_{c}} \max _{\mathbf{d} \in \tilde{\mathcal{D}}} \frac{\mathbf{w}^{T} \mathbf{d}}{\left\|\mathbf{w}_{\mathcal{T}}\right\|\|\mathbf{d}\|}=\inf _{\mathbf{w} \in \mathcal{R}_{c}} \max _{\mathbf{d} \in \tilde{\mathcal{D}}} \frac{\mathbf{w}^{T} \mathbf{d}}{\|\mathbf{w}\|\|\mathbf{d}\|} \geq \frac{\alpha}{n} .
$$

So we set $\kappa_{2 c}=\alpha / n$. To finish the treatment of this case, define $\kappa_{2}$ and note

$$
0<\kappa_{2}=\min \left\{\kappa_{2 a}, \kappa_{2 b}, \kappa_{2 c}\right\} \leq \inf _{\substack{\mathbf{w} \in \mathbb{R}^{n} \\ \mathbf{w} \mathcal{T} \neq 0}} \max _{\mathbf{d} \in\{\tilde{\mathcal{D}}, \tilde{\mathcal{C}}\}} \frac{\mathbf{w}^{T} \mathbf{d}}{\left\|\mathbf{w}_{\mathcal{T}}\right\|\|\mathbf{d}\|}=\kappa(\{\tilde{\mathcal{D}}, \tilde{\mathcal{C}}\}) .
$$

$|\mathcal{I}|>1$ (multiple active constraints) $\tilde{\mathcal{C}}$ positively spans $\partial \mathcal{T}$ by definition. Invoking Proposition 1 (Appendix A) shows that it also positively spans $\mathcal{T}$ in this case. Adding vectors in the interior of the tangent cone does not change this, so $\{\tilde{\mathcal{D}}, \tilde{\mathcal{C}}\}$ must generate $\mathcal{T}$. For each combination of active constraints, we have one and only one $\tilde{\mathcal{C}}$. We take $\kappa_{3}$ to be the minimum of $\kappa(\tilde{\mathcal{C}})$ over this finite set.

To conclude, note that in all three cases $\{\tilde{\mathcal{D}}, \tilde{\mathcal{C}}\}$ generates $\mathcal{T}$ and the cosine measure $\kappa(\{\tilde{\mathcal{D}}, \tilde{\mathcal{C}}\})$ can be bounded below by a strictly positive constant - i.e., $\kappa_{m i n}$. This constant is independent of the iteration $k$.

6.2 Conditions on the step lengths, the stencil contraction factor and the forcing function

With Lemma 11 we have shown that the set of search directions is rich enough to find the existing feasible descent directions. It is also conditioned well in the sense that the angle between the descent direction and one of the search directions is never too large. If the steps taken along these directions are too small or too large, though, the optimization might still fail to converge 27, 35. The following results assure that this is not the case.

Lemma 2 (28, Condition 2). There exist $\beta_{\max } \geq \beta_{\min }>0$, independent of $k$, such that for every $k$ for which $\mathcal{T}\left(\mathbf{x}_{k}, \epsilon_{k}\right) \neq\{\mathbf{0}\}$, the following holds:

$$
\beta_{\min } \leq\|\mathbf{d}\| \leq \beta_{\max } \quad \forall \mathbf{d} \in\{\tilde{\mathcal{D}}, \tilde{\mathcal{C}}\}_{k}
$$

In the algorithms described here, we simply normalize all search directions which immediately leads to Lemma 2, But the condition is less strict than this: uniform bounds on the vector norms are enough. This leaves open many possibilities for dynamic scaling of the search directions, which is likely to be an effective technique for speeding up convergence [33, 28, 9, 35.

Lemma 3 (analogue of 28, Condition 3). For all $\mathbf{d}_{k}^{(i)} \in\{\tilde{\mathcal{D}}, \tilde{\mathcal{C}}\}_{k}$, the associated trial step is of the form $\tilde{\delta}_{k}^{(i)} \mathbf{d}_{k}^{(i)}$ with $\tilde{\delta}_{k}^{(i)} \in\left[0 ; \delta_{k}\right]$. In addition, the individual step lengths $\tilde{\delta}_{k}^{(i)}$ are as long as possible while respecting $\mathbf{x}_{k}+\tilde{\delta}_{k}^{(i)} \mathbf{d}_{k}^{(i)} \in \Omega$.

The validity of the lemma is obvious from the working principles of the algorithms. The individual step lengths $\tilde{\delta}_{k}^{(i)}$ may be reduced (to move onto the boundary), but the longest possible step is always taken.

Lemma 4 ([28, Conditions 4 and 5). The forcing function $\rho(t)$ satisfies following criteria:

1. $\rho(t)$ is continuous for $t \geq 0$. 
2. $\lim _{t \downarrow 0} \rho(t) / t=0$.

3. $\rho\left(t_{1}\right) \leq \rho\left(t_{2}\right)$ if $t_{1} \leq t_{2}$.

4. $\rho(t)>0$ for $t>0$

As the forcing function is an input to Algorithm 1 , it is the user's responsibility to ascertain that this condition is respected. The functions suggested in Section 4 are valid options, though. The choice $\rho(t)=\tau_{\text {tol }} t^{2}$ with $\tau_{\text {tol }}>0$ is conventional. In this case, the properties are easy to deduce. If the user opts to implement equation (18), the lemma also holds under the additional assumption that the objective function is bounded below on the feasible set. Let $f_{*}$ represent such a lower bound. Then, for all $k$,

$$
\tau_{\text {tol }} t^{2} \leq \rho_{k}(t) \leq \max \left(1,\left|f\left(\mathbf{x}_{0}\right)\right|,\left|f_{*}\right|\right) \tau_{\text {tol }} t^{2} .
$$

Hence, $\rho_{k}(t)=O\left(t^{2}\right)$ with an iteration-independent constant. The fact that the coefficient in the monomial can vary over the iterations $k$ does not interfere with the convergence proofs in 28]: global convergence is still assured because the coefficient is bounded away from zero; the stationarity result $\left(\liminf _{k \rightarrow \infty} \chi\left(\mathbf{x}_{k}\right)=0\right)$ still holds because the coefficient is bounded above.

The next lemma is a trivial property of the algorithms presented here (because $\theta_{k}=\theta$ by the definition of Algorithm 1] but it is technically necessary to prove the global convergence.

Lemma 5 ([28, Condition 6$)$. A constant $\theta_{\max }<1$ exists such that $\theta_{k} \leq \theta_{\max }$ for all $k$.

Finally, there is another remark to make. The previous lemmas correspond to the six formal conditions put forth by Kolda et al. [28]. However, the convergence error bound of Theorem 4 requires an additional assumption that is less emphasized in the previous work but is still important. The assumption made there is that the parameter $\epsilon_{k}$, used to qualify constraints as active or inactive, is tied directly to the step length parameter as $\epsilon_{k} \equiv \beta_{\max } \delta_{k}$. It turns out that the above assumption is a bit conservative: it may be relaxed as follows without destroying the convergence property.

Lemma 6. A constant $\epsilon_{\text {tol }}>0$ exists such that $\epsilon_{k}=\epsilon_{\text {tol }} \beta_{\max } \delta_{k}$ for all $k$.

It is clear from the algorithm definitions that the above statement is true. The reason why the relaxation is possible is that Lemma 6, together with Lemma 2 and 3 , guarantees that all of the steps along the core search directions will be both feasible and of length greater than $\epsilon_{\mathrm{tol}} \beta_{\max } \delta_{k}$. Readers interested in more detail may consult the proofs of [28], Theorems 6.3 and 6.4. Our motivation for applying the relaxed assumption is that setting $\epsilon_{\mathrm{tol}}<1$ will prevent a search direction update that is too aggressive, i.e. that takes into account boundaries that are still relatively far away.

\section{Numerical examples}

In the following section, we test if the adaptive approaches described in the previous part have a real advantage over other direct search methods, both for a classical benchmark problem set and for a realistic engineering application. Unless other values are specified, the parameters of GIGS are the default given in Table $2{ }^{1}$

\footnotetext{
1 All treated problems are fully bounded, so the default value for $\delta_{0}$ is defined and finite.
} 
Table 2: Default parameter settings for Algorithm 1

\begin{tabular}{ll} 
Parameter & Value \\
\hline$\delta_{0}$ & $\max \left\{1, \max _{i}\left|b_{u}^{(i)}-x_{0}^{(i)}\right|, \max _{i}\left|b_{l}^{(i)}-x_{0}^{(i)}\right|\right\}$ \\
$\delta_{\text {tol }}, \epsilon_{\text {tol }}$ & $10^{-9}$ \\
$\theta$ & 0.5 \\
$\phi$ & 1 \\
$\rho$ & $\sqrt[18]{ }$ with $\tau_{\text {tol }}=10^{-6}$ \\
$q, r$ & {$[\sqrt{n}\rfloor$}
\end{tabular}

\subsection{Academic test problems}

The popular test collection of Moré and Wild [34], tailored towards derivative-free optimization algorithms, is taken as a starting point. This set consists of 53 nonlinear least-squares problems of varying complexity, with design dimensions ranging between 2 and 12 . As explained in [34], these problems can be easily modified to become nonsmooth and/or to emulate numerical noise.

The problems in Moré and Wild's set come with well-defined starting points $\mathbf{x}_{0}$, but are free of constraints 34. To increase their representativity of the constrained problems targeted here, we introduce some (nontrivial) bounds on the design variables. As detailed in Appendix B, these are chosen such that the new solution is constrained to a varying degree.

The testing procedure as a whole also follows the standard practice of 34. Each of the problems in the set is passed as input to the different solvers. Very tight convergence tolerances $\left(\delta_{\mathrm{tol}}=10^{-9}\right)$ are prescribed to force sufficiently long iteration histories. The cost of the optimization is expressed in (multiples of) objective function evaluations: this is assumed to be the major component in the total operation count for $\mathrm{BBO} / \mathrm{SBO}$ and is independent of the particular computer system and code implementation. To judge and compare the performance we also use the typical metric for DFO,

$$
\tau=\frac{f_{k}-f_{*}}{f_{0}-f_{*}}
$$

which measures the relative progress of the best function value in iteration $k$ towards the optimal value. After completion of all the tests, the results can be graphically represented with data profiles 34 .

Figures 1, 2 and 3 compare different variants of GIGS. The figures respectively show the profiles for the smooth, noisy and nondifferentiable problem sets. For each set, two graphs are shown. The upper graph uses a more relaxed convergence threshold of $\tau=10^{-2}$ and the lower one is stricter in declaring $\tau=10^{-4}$.

Each of the graphs shows a similar general trend. It seems that the relative performance of the proposed algorithms is only weakly dependent on the considered problem type. The similarities are particularly large between Figures 1 and 2, as expected, GSS methods remain fairly undisturbed by noise on the objective function. While the introduction of nonsmoothness and the smaller convergence tolerances cause a smaller fraction of problems to be solved, the ranking of the methods is not drastically altered.

For all problem sets and tolerances, we observe that the profiles of the opportunistic (O) GIGS variants almost immediately diverge from those using complete polling $(\mathrm{C})$. The former group always appears more efficient, while robustness - indicated by the behavior for larger computational budgets - is comparable.

Because the sensitivity information is accumulated over several (unsuccessful) iterations, the differences between the adaptive methods (variants 3 to 6 ) and the more classical approaches 
(variants 1 to 2 ) starts to become apparent after expending around 10 to 20 budget units. After this point, the adaptive variants generally seem more efficient; compare for instance the profiles of Algorithms 1, 1 and 1,2 to their adaptive counterparts, Algorithms 1,5 and 1,6. While the data profiles of Algorithms 1,4 and 1, 6 look very similar, upon closer inspection we find that variant 1.6 performs slightly better on average. This version would be our first recommendation for practical use.

We conclude the academic benchmark tests by comparing the (opportunistic, adaptive) GIGS method to two state-of-the-art methods that also combine concepts of GSS and simplex derivatives. The first method is the Matlab implementation (v0.3) of implicit filtering [26], available via https://ctk.math.ncsu.edu/imfil.html. We set this method to use a steepest-descent line search. Second, we use the method of [16] (SID-PSM); the Matlab implementation (v1.3) of this method was provided by the original authors. We make SID-PSM use the coordinate search directions and omit the model-based search step described in [15. Figure 4 shows the comparison of the three algorithms for the aggregated problem set, which envelops the smooth, noisy, and nondifferentiable instances of the academic problems. In the test, GIGS competes well with both state-of-the-art methods.
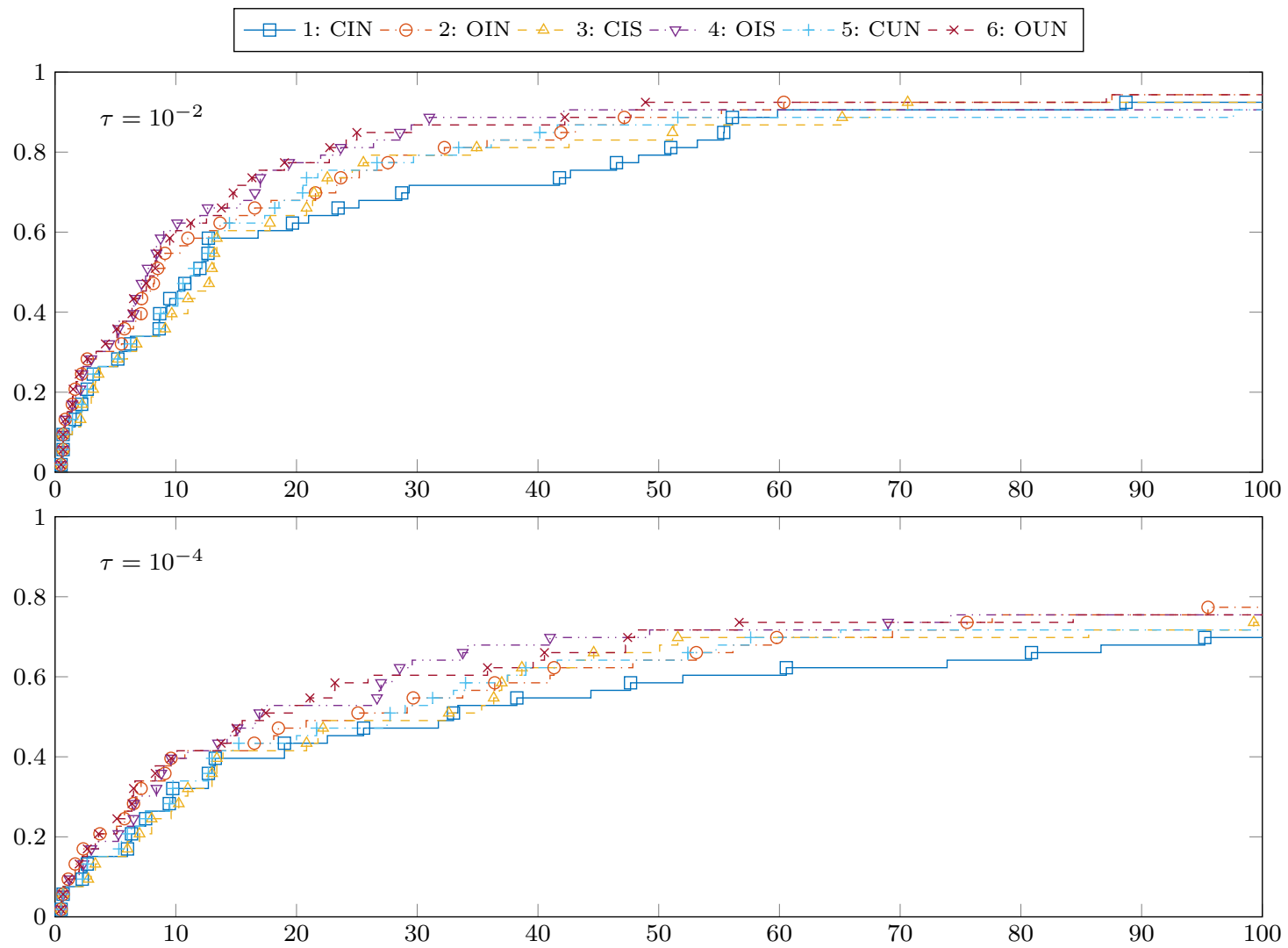

Fig. 1: Data profiles for smooth problems. The variants of Algorithm 1 are compared for two different tolerances $\tau$. Cost is measured in number of simplex gradient equivalents. The legend entries correspond to the classification in Table 1 . 

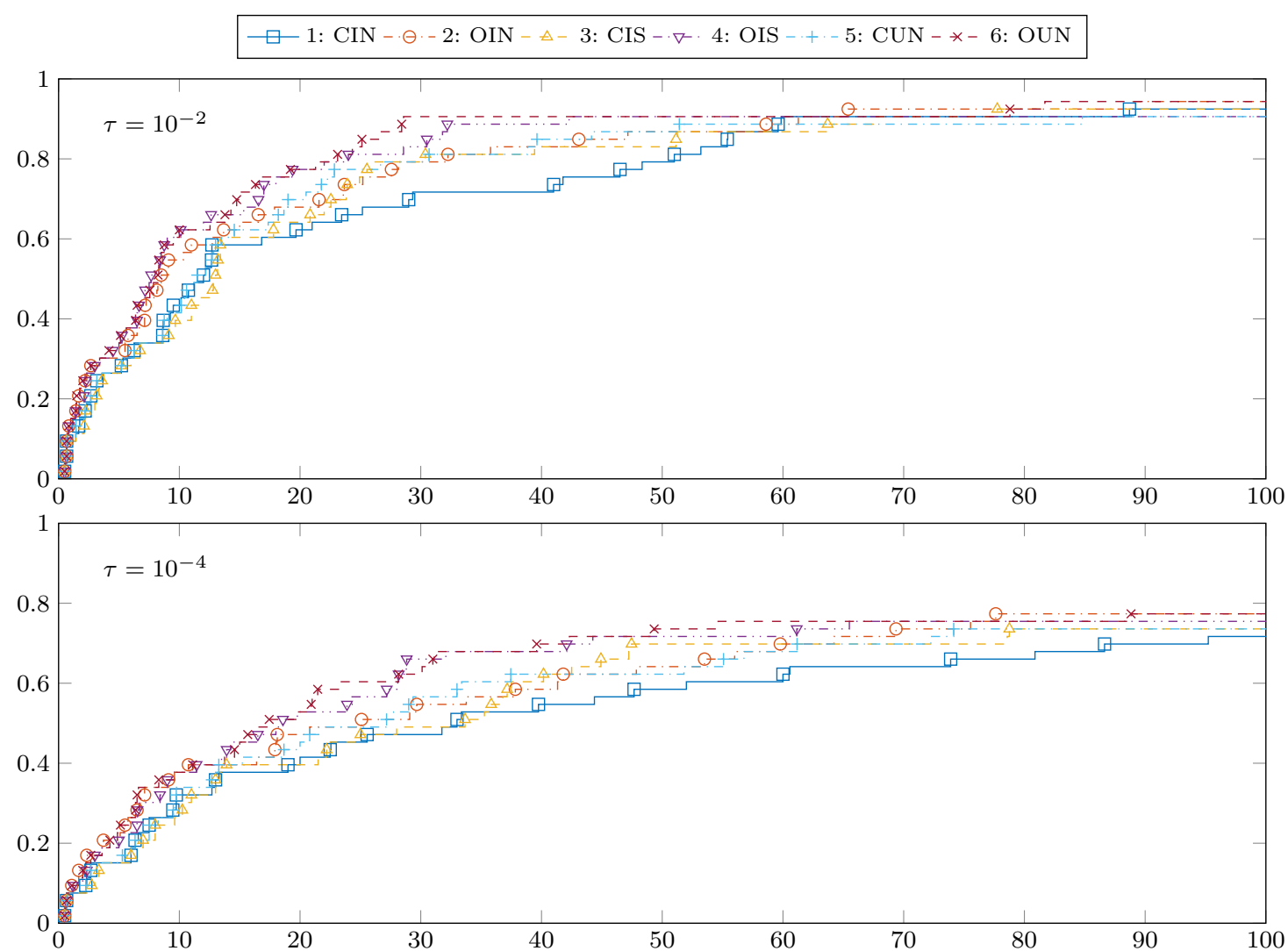

Fig. 2: Data profiles for noisy problems. The variants of Algorithm 1 are compared for two different tolerances $\tau$. Cost is measured in number of simplex gradient equivalents. The legend entries correspond to the classification in Table 1.

7.2 Engineering case study: multidisciplinary design optimization of a black-box mechatronics system

As argued in the introduction, the further development of DFO, BBO and SBO is motivated in large part by challenges arising in industrial practice. An industrially relevant optimization problem may involve many heterogeneous design variables and rely on responses generated from complex, coupled and expensive simulations. The implementation will typically consist of a patchwork of different software tools based on propriety or legacy codes, making analysis beyond the black-box level practically impossible. The following problem is supposed to be a reflection of this reality.

We consider a simulation model representing a quarter-car system with a mechatronic, active suspension (Figure 5). The system consists of a rigid four-bar linkage, mechanically supported by a spring, a nonlinear damper and a controlled actuator. The leftmost bar is attached to the car body, whose motion is restrained to a vertical translation, and the rightmost bar represents the wheel hub, which receives a position input signal. The goal is to find a design that minimizes the peak acceleration of the car body when the wheel hub experiences a stepwise upward motion. Besides peak acceleration, the objective function includes a term to penalize suspensions that are too soft to support the car in the static case. The available design parameters are as follows: 

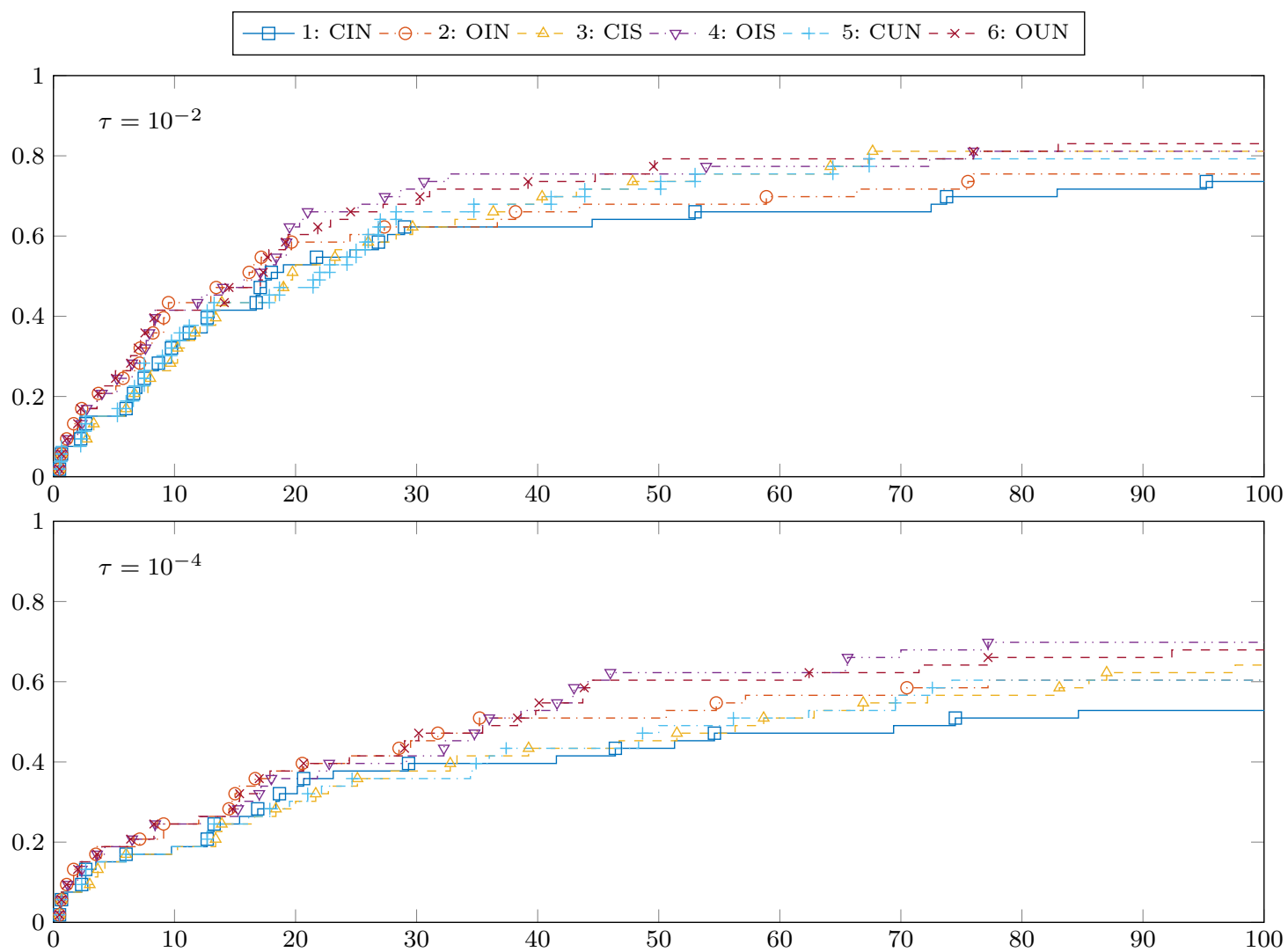

Fig. 3: Data profiles for nondifferentiable problems. The variants of Algorithm 1 are compared for two different tolerances $\tau$. Cost is measured in number of simplex gradient equivalents. The legend entries correspond to the classification in Table 1.

4 lengths and an angle defining the geometry of the linkage, the spring constant, 3 parameters governing the nonlinear damper, and 2 gains of a PI controller which translates the body acceleration to the actuator force. In total, this yields an 11-dimensional design space, which is bounded by the values in Table 3 .

The model was implemented in the graphical environment Simulink, so the details of its workings are unknown and simulation is automated to a large degree. The interface between the objective function and the optimization algorithm thus stays limited to simple evaluations: a particular design point leads to a particular peak acceleration. Aside from the bounds, all constraints are hidden to the user. In-depth considerations of the input parameter's internal consistency (e.g. geometrical compatibility) are not part of the a-priori formulation, so even for points inside the feasible region $\Omega$ these evaluations may not return a meaningful output within a predictable time frame. All simulations that exceeded a certain time window or failed otherwise were set to return a non-numerical failure indicator.

For the numerical optimization, the design variables were rescaled, linearly mapping the feasible set onto the hyperrectangle $[-1 ; 1]^{n}$. The zero vector was used as a starting point, the expansion parameter $\phi$ was changed to 2 in order to promote exploration, and the tolerances $\delta_{\text {tol }}$ and $\tau_{\text {tol }}$ were relaxed to $10^{-2}$ and $10^{-4}$ respectively. All other settings take the default values from Table 2 , 

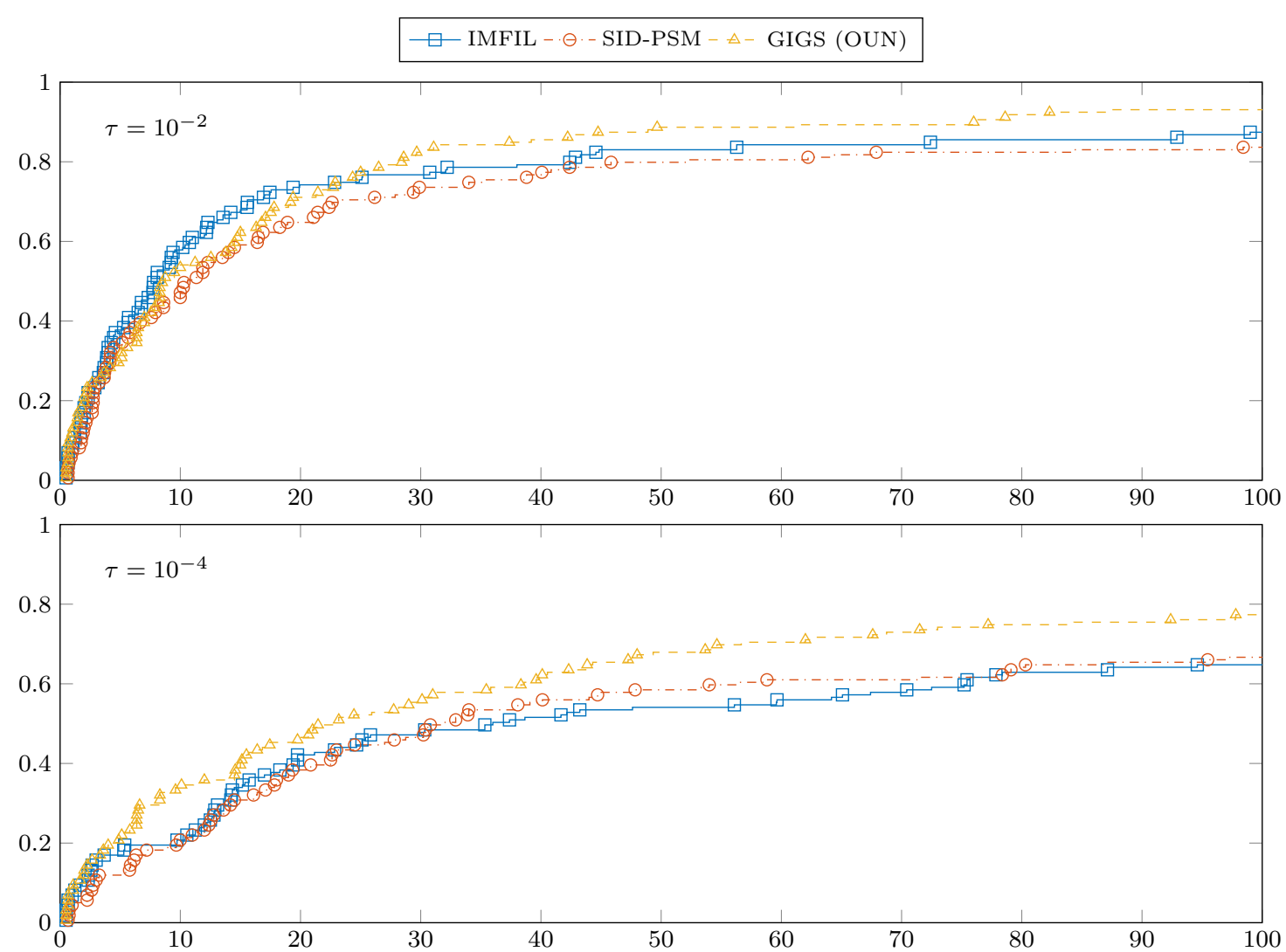

Fig. 4: Data profiles for aggregated smooth, noisy, and nondifferentiable problems. The variant OUN of Algorithm 1 is compared to two other methods for two different tolerances $\tau$. Cost is measured in number of simplex gradient equivalents.

Table 3: Descriptions and bounds for the design variables.

\begin{tabular}{lcc}
\hline Description & Symbol & Range \\
\hline Inclination of the body link & $\alpha$ & $0^{\circ}-60^{\circ}$ \\
Length of the body link & $l_{1}$ & $0.1-0.3 \mathrm{~m}$ \\
Vertical wheel-body distance & $l_{2}$ & $0.1-0.3 \mathrm{~m}$ \\
Horizontal wheel-body distance & $l_{3}$ & $0.2-0.4 \mathrm{~m}$ \\
Length of the wheel link & $l_{4}$ & $0.2-0.4 \mathrm{~m}$ \\
Spring stiffness & $k$ & $10^{4}-10^{5} \mathrm{Nm}^{-1}$ \\
Low-speed damping coefficient & $c_{1}$ & $10^{4}-10^{5} \mathrm{Nm}^{-1} \mathrm{~s}$ \\
High-speed damping coefficient & $c_{2}$ & $5 \times 10^{3}-5 \times 10^{4} \mathrm{Nm}^{-1} \mathrm{~s}$ \\
Damping speed threshold & $b$ & $0-1 \mathrm{~ms}^{-1}$ \\
Proportional controller gain & $K_{P}$ & $0-10^{3}$ \\
Integral controller gain & $K_{I}$ & $0-10^{3}$ \\
\hline
\end{tabular}




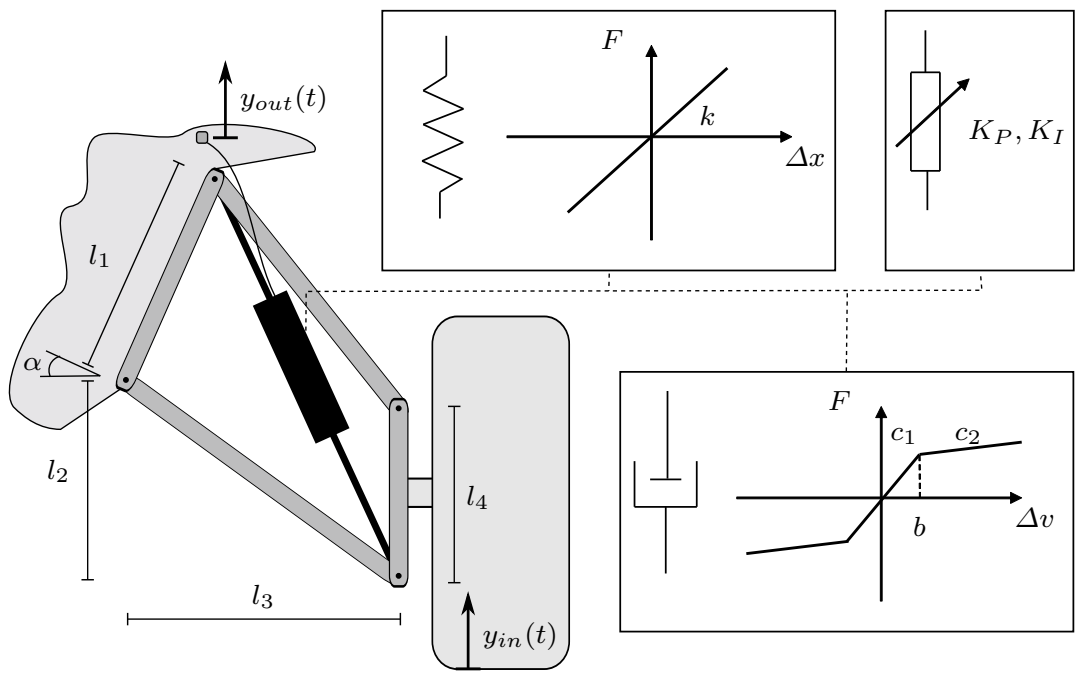

Fig. 5: Model and design parameters for the quarter-car with an active suspension consisting of a spring, nonlinear damper and PI-controlled actuator. The configuration depicts a static equilibrium.

The convergence plot in Figure 6 shows the performance metric $\sqrt{31}$ as a function of the number of function evaluations. After 1000 evaluations, all but two optimization trajectories have approached a similar design point - the fact that some algorithms eventually find better regimes is, in our view, more likely a stroke of luck than a consequence of any particular methodical feature. However, the results provide confirmation that the GSS paradigm is robust enough to tackle challenging simulation-based problems, and that this property is not compromised by introducing the adaptation strategy. The speed of convergence, on the other hand, differs significantly between all implementations. The characteristic L-shape can be easily discerned, but is more pronounced for opportunistic Algorithms 1,2, 1,4 and 1, 6 .

It is more difficult to draw conclusions about the effects of involving simplex gradients, since some variants perform better and others worse than the non-adaptive reference. Several reasons could be brought up to explain this. First, the minimum is found fairly quickly so the gradient accumulation has not that much time to really come into effect and make a difference. The function evaluation count includes failed simulations, which occurred regularly, so the actually useful output is limited. It also seems that in this test case, having canonical vectors among the search directions is a more important factor. Possibly this is due to the heterogeneity of the design parameters, some of which have a much more pronounced impact on the model performance.

\section{Conclusion}

In this work, we describe gradient-informed generating set search algorithms for solving minimization problems whose objective and constraints are general black-boxes, possibly derived from numerical simulations. These applications often involve extreme types of nonsmoothness in the form of software errors and numerical noise that cause classical solvers to fail. Generating set searches, on the other hand, can be shown to converge even for problems lying far from the smooth ideal. The GIGS algorithms aim to exploit this established robustness, but strive for better efficiency by incorporating a novel strategy for selecting the search directions. This is done 


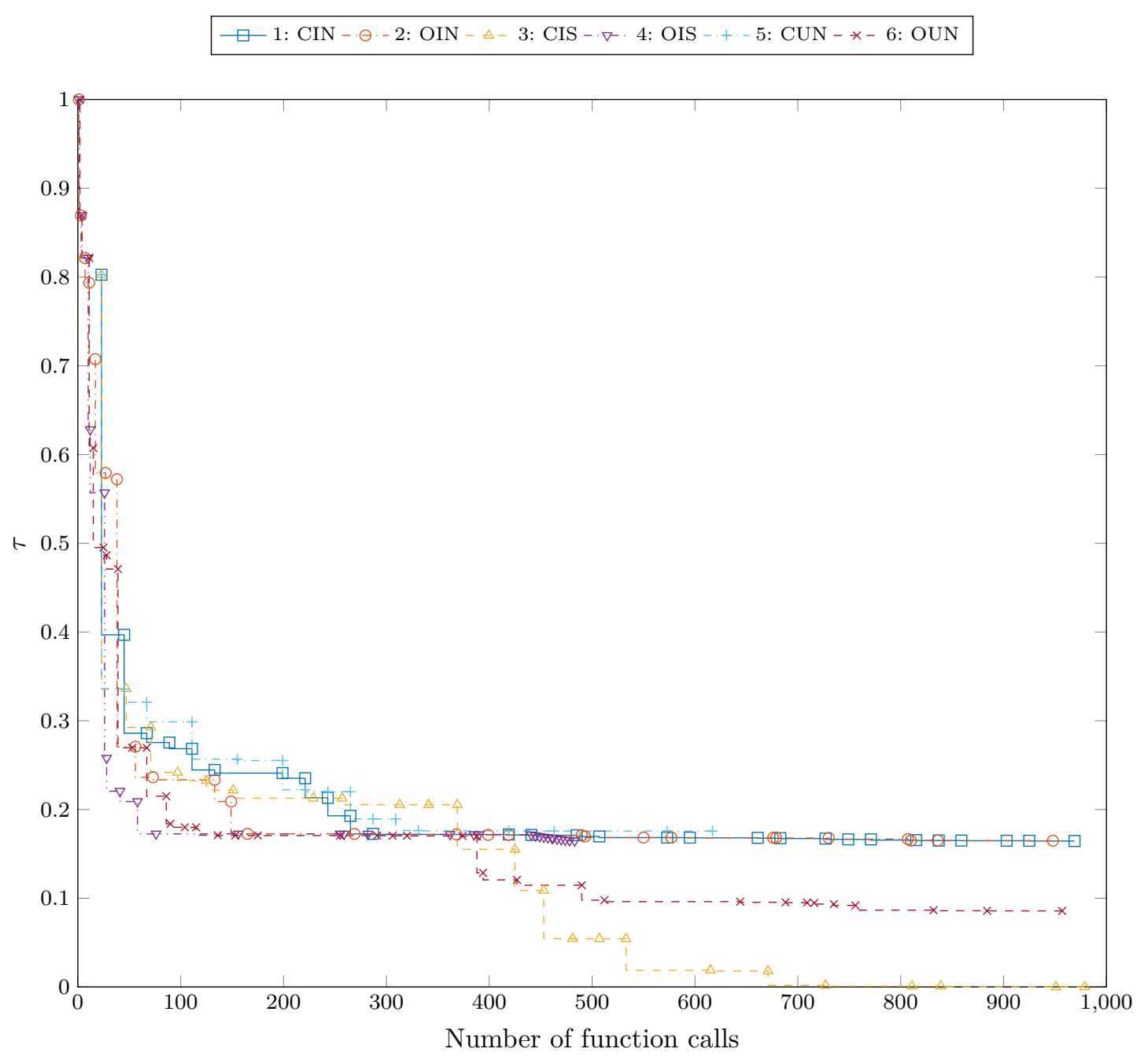

Fig. 6: Convergence plots for the quarter-car optimization problem. The graph shows relative progress towards the best known objective value $(\tau)$ versus the number of function calls (including failures) for the six different variants of Algorithm 1. Cost is measured in number of objective function evaluations. The legend entries correspond to the classification in Table 1

by accumulating simplex gradient estimates and aligning the search stencil with the principal components of the sample. Because no additional function evaluations are required, the added expense is small. Several concrete implementation schemes are proposed and tested. We prove that they are all valid instances of GSS, thus inheriting the extensive first-order convergence guarantees. Additionally, the adaptive rotation of the search stencil is expected to improve the confidence of finding solutions satisfying second-order optimality criteria. To test the efficiency in practice, numerical experiments are performed on both an academic benchmark set and an engineering case study, consisting of the design optimization of an actively suspended quartercar. The tests show that an opportunistic polling strategy is usually most effective in reducing the number of function evaluations required to find a local optimum. In most cases, the adaptive choice of search directions led to significant speed-ups in comparison with the non-adaptive ref- 
erence and in comparison with two other direct search methods. In future work, further efficiency gains could be pursued, for instance by introducing individual step length rescaling, adaptively reducing the stencil dimension, reflecting simplex gradient information in the stencil geometry, or exploiting cached function values. Potential extensions to multi-objective optimization problems could also be investigated.

\section{Acknowledgments}

We kindly thank prof. A.L. Custódio for providing us with an implementation of the SID-PSM algorithm. We also thank two anonymous referees for their constructive input in preparing this article. Our work was supported by the Research Fund KU Leuven and by Flanders Make, the strategic research center for the manufacturing industry. The research of F. Naets is funded by a grant from the Research Foundation - Flanders (FWO). 


\section{A Polyhedral cones and their application to linearly constrained optimization}

A polyhedral cone $\mathcal{L}$ in $\mathbb{R}^{n}$ is the set of vectors satisfying linear inequalities in the set $\mathcal{A}$,

$$
\mathcal{L}=\left\{\mathbf{w} \mid \mathbf{a}^{T} \mathbf{w} \leq 0, \forall \mathbf{a} \in \mathcal{A}\right\}
$$

Unless the a are all zero vectors, the cone can be seen as an intersection of half-spaces whose bounding hyperplanes are determined by the normal vectors a. The boundary of the cone $\partial \mathcal{L}$ is then the set of points lying on at least one of these hyperplanes

$$
\partial \mathcal{L}=\left\{\mathbf{w} \in \mathcal{L} \mid \exists \mathbf{a} \in \mathcal{A} \backslash\{\mathbf{0}\}: \mathbf{a}^{T} \mathbf{w}=0\right\}
$$

(all other points of $\mathcal{L}$ are in its interior). The polar of the cone $\mathcal{L}$ is defined as follows:

$$
\mathcal{L}^{\circ}=\left\{\mathbf{w} \mid \mathbf{y}^{T} \mathbf{w} \leq 0, \forall \mathbf{y} \in \mathcal{L}\right\}
$$

We denote the (orthogonal) projection of a vector $\mathbf{w}$ onto $\mathcal{L}$ by $\mathbf{w}_{\mathcal{L}}$; if $\mathbf{w}_{\mathcal{L}}=\mathbf{0}$ then $\mathbf{w} \in \mathcal{L}^{\circ}$ and vice versa. Moreover, any vector in $\mathbb{R}^{n}$ can be decomposed as 28

$$
\mathbf{w}=\mathbf{w}_{\mathcal{L}}+\mathbf{w}_{\mathcal{L}^{\circ}} \quad \text { with } \quad \mathbf{w}_{\mathcal{L}}^{T} \mathbf{w}_{\mathcal{L}^{\circ}}=0 .
$$

Applying this in the context of linearly constrained optimization in $\mathbb{R}^{n}$, the set $\mathcal{A}$ is represented by the rows of the constraint matrix $\mathbf{A}$, labeled with an index $i$. For a (feasible) point $\mathbf{x}$ in the design space $\Omega$ (see Equation (20), the $\epsilon$-active constraints are defined by

$$
\mathcal{I}(\mathbf{x}, \epsilon)=\left\{i\left|\mathbf{b}_{i}-\mathbf{a}_{i}^{T} x \leq \epsilon\right| \mid \mathbf{a}_{i} \|\right\},
$$

where $\epsilon$ is a positive number. These are all the constraints whose boundary is within a distance $\epsilon$ from $\mathbf{x}$. The $\epsilon$-tangent cone then comprises all steps from $\mathbf{x}$ that are not pointing towards any of the $\epsilon$-active constraints:

$$
\mathcal{T}(\mathbf{x}, \epsilon)=\left\{\mathbf{w} \mid \mathbf{a}_{i}^{T} \mathbf{w} \leq 0, \forall i \in \mathcal{I}(\mathbf{x}, \epsilon)\right\} .
$$

A polyhedral cone can equivalently be seen as the set of positive (conical) linear combinations of a number of vectors, called the generators of the cone. It is therefore said to be finitely generated, and we denote the cone $\mathcal{L}$ generated by the set $\mathcal{Y}$ as $\mathcal{L}(\mathcal{Y})$. The following general result (e.g. [20, Theorem 16) will help in the development.

Proposition 1. For finitely generated cones, the following holds:

1. In case $\mathcal{L} \subset \mathbb{R}^{n}$ is not equal to a half-space, a set $\mathcal{Y}$ of vectors positively spans the cone $\mathcal{L}$ if it positively spans $\partial \mathcal{L}$.

2. In case $\mathcal{L} \subset \mathbb{R}^{n}$ is a half-space of $\mathbb{R}^{n}$, a set $\mathcal{Y}$ of vectors positively spans the cone $\mathcal{L}$ if it positively spans $\partial \mathcal{L}$ and it includes at least one vector in the interior of $\mathcal{L}$.

3. In case $\mathcal{L}=\mathbb{R}^{n}$, the boundary $\partial \mathcal{L}$ is empty. function

In $\mathbb{R}^{n}$, given some cone $\mathcal{L} \neq\{\mathbf{0}\}$ and some finite set $\mathcal{Y} \neq\{\mathbf{0}\}$, we define their mutual cosine measure as the

$$
\tilde{\kappa}(\mathcal{L}, \mathcal{Y}) \equiv \inf _{\substack{\mathbf{w} \in \mathbb{R}^{n} \\ \mathbf{w}_{\mathcal{L}} \neq 0}} \max _{\mathbf{y} \in \mathcal{Y}} \frac{\mathbf{w}^{T} \mathbf{y}}{\left\|\mathbf{w}_{\mathcal{L}}\right\|\|\mathbf{y}\|} .
$$

In case $\mathcal{L}=\mathcal{L}(\mathcal{Y})$, this becomes identical to the quantity $\kappa(\mathcal{Y})$ given in [28], i.e.

$$
\kappa(\mathcal{Y}) \equiv \tilde{\kappa}(\mathcal{L}(\mathcal{Y}), \mathcal{Y}) .
$$

It was shown, for instance by Lewis et al. [32], that $\kappa(\mathcal{Y})>0$.

\section{B Details of the academic test problems}

The academic benchmark set is derived from that of Moré and Wild 34. Through changing the dimension $n$ and multiplying the given starting point $\mathbf{x}_{0}$ by an additional factor, 22 named functions lead to a set of 53 problems, as in the original benchmark. We add constraints as follows. First, a global reference solution $\mathbf{x}_{*}$ was found for all but three problems, either through analytical methods, referencing other works or by extensive empirical testing (Table 4). The precise values of the coordinate bounds are then chosen in one of three ways. For a first group of problems, we ensure that the solution will be maximally constrained by setting 


$$
b_{l}^{(i)}=x_{0}^{(i)}-\left|x_{*}^{(i)}-x_{0}^{(i)}\right|, \quad b_{u}^{(i)}=x_{0}^{(i)}+\left|x_{*}^{(i)}-x_{0}^{(i)}\right|
$$

for all components $i$. A more mildly constrained solution is obtained using the formula

$$
b_{l}^{(i)}=x_{0}^{(i)}-\max _{j}\left\{\left|x_{*}^{(j)}-x_{0}^{(j)}\right|\right\}, \quad b_{u}^{(i)}=x_{0}^{(i)}+\max _{j}\left\{\left|x_{*}^{(j)}-x_{0}^{(j)}\right|\right\} .
$$

In the third case we select the bounds some distance away from the reference solution (ad hoc). The final result is that the original reference solution $\mathbf{x}_{*}$ remains in the feasible domain, with either all, some or no bounds in the new active constraint set. In assigning the problems to each of these three cases, we aim for a more or less even distribution, details of which can be found in Table 4 For 10 functions giving 18 problems, the optimum is fully constrained. For 9 functions giving 17 problems, the optimum is moderately constrained. For 3 functions giving 18 problems, the optimum is unconstrained.

The starting point for the Mancino function is given by the formula

$$
x_{0}^{(i)}=-8.711 \times 10^{-4}\left\{(i-50)^{3}+\sum_{j=1}^{n} \sqrt{i / j}\left[\sin ^{5}(\log (\sqrt{i / j}))+\cos ^{5}(\log (\sqrt{i / j}))\right]\right\} .
$$

For the rank-1 linear functions, an exact solution can be found as

$$
x_{*}^{(i)}=3 i\left(15 \sum_{j=1}^{n} j^{2}\right)^{-1}
$$

or

$$
\begin{aligned}
& x_{*}^{(1)}=0, \quad x_{*}^{(n)}=0, \\
& x_{*}^{(i)}=3 i\left(11 \sum_{j=2}^{n-1} j^{2}\right)^{-1} \text { for } i \in\{2, \ldots, n-1\}
\end{aligned}
$$

The 'bdqrtic' function has a dimension-dependent solution that is not available in closed form. We use the following empirical values:

$$
\begin{array}{ll}
\mathbf{x}_{*}=(0.6161,0.4862,0.3919,0.3264,0,0,0,0) & \text { if } n=8 \\
\mathbf{x}_{*}=(0.6255,0.4851,0.3672,0.2852,0.3302,0.3776,0,0,0,0) & \text { if } n=10 \\
\mathbf{x}_{*}=(0.6251,0.4858,0.3713,0.2835,0.3169,0.3387,0.3759,0,0,0,0) & \text { if } n=11 \\
\mathbf{x}_{*}=(0.6248,0.4854,0.3717,0.2860,0.3155,0.3254,0.3378,0.3740,0,0,0,0) & \text { if } n=12
\end{array}
$$

\section{References}

1. Abramson, M.A.: Second-order behavior of pattern search. SIAM Journal on Optimization 16(2), 515-530 (2005)

2. Abramson, M.A., Audet, C.: Convergence of mesh adaptive direct search to second-order stationary points SIAM Journal on Optimization 17(2), 606-619 (2006)

3. Abramson, M.A., Brezhneva, O.A., Dennis, J.E., Pingel, R.L.: Pattern search in the presence of degenerate linear constraints. Optimization Methods and Software 23(3), 297-319 (2008)

4. Abramson, M.A., Frimannslund, L., Steihaug, T.: A subclass of generating set search with convergence to second-order stationary points. Optimization Methods and Software 29(5), 900-918 (2014)

5. Audet, C.: A survey on direct search methods for blackbox optimization and their applications. In: P.M. Pardalos, T.M. Rassias (eds.) Mathematics Without Boundaries, pp. 31-56. Springer, New York (2014)

6. Audet, C., Dennis, J.E.: Analysis of generalized pattern searches. SIAM Journal on Optimization 13(3), 889-903 (2003)

7. Audet, C., Dennis, J.E.: Mesh adaptive direct search algorithms for constrained optimization. SIAM Journal on Optimization 17(1), 188-217 (2006)

8. Audet, C., Hare, W.: Derivative-Free and Blackbox Optimization, 1st edn. Springer Series in Operations Research and Financial Engineering. Springer, Cham (2017)

9. Audet, C., Le Digabel, S., Tribes, C.: Dynamic scaling in the mesh adaptive direct search algorithm for blackbox optimization. Optimization and Engineering 17(2), 333-358 (2016) 
Table 4: Starting points, reference solutions and bounds for the academic problems.

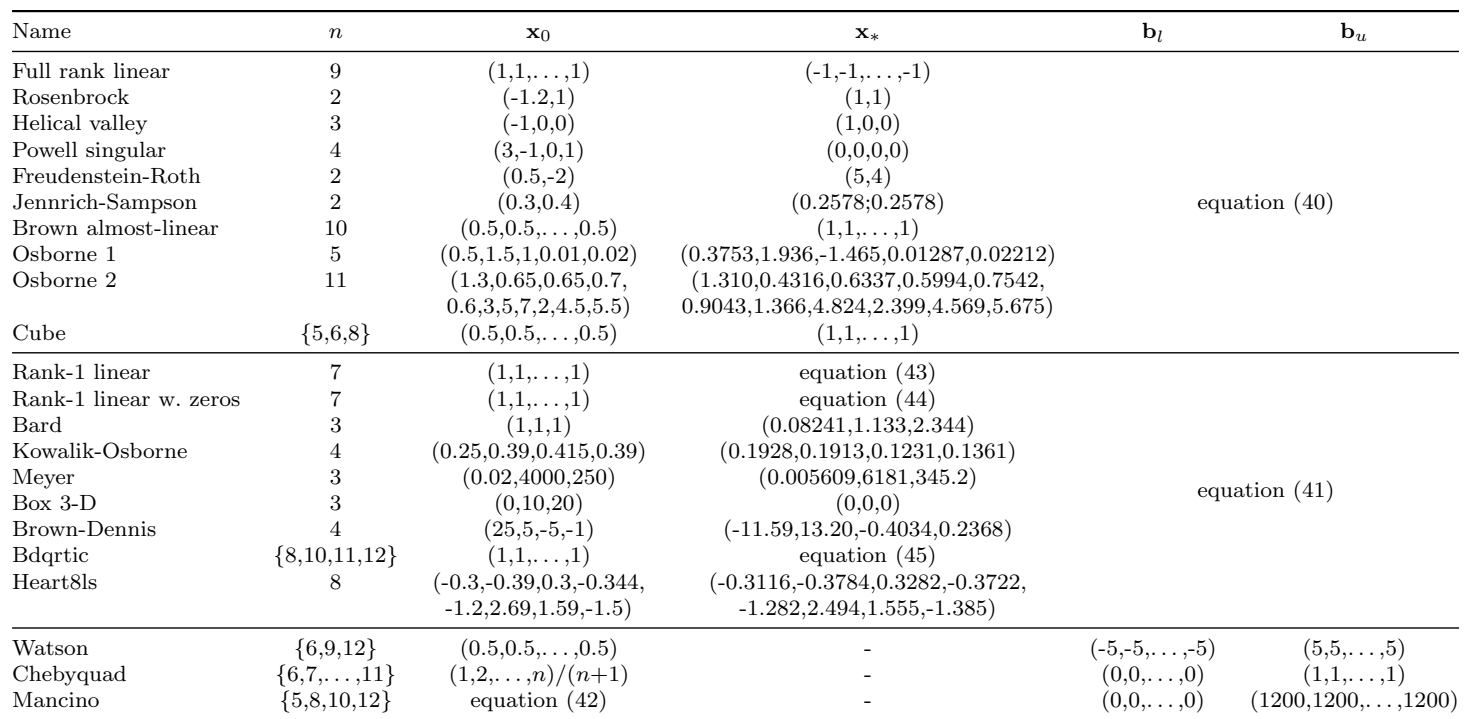

10. Bortz, D.M., Kelley, C.T.: The simplex gradient and noisy optimization problems. In: J. Borggaard, J. Burns, E. Cliff, S. Schreck (eds.) Computational Methods for Optimal Design and Control, pp. 77-90. Birkhäuser, Boston (1998)

11. Brand, M.: Fast low-rank modifications of the thin singular value decomposition. Linear Algebra and its Applications 415(1), 20-30 (2006)

12. Conn, A.R., Scheinberg, K., Vicente, L.N.: Geometry of sample sets in derivative-free optimization: Polynomial regression and underdetermined interpolation. IMA Journal of Numerical Analysis 28(4), 721-748 (2008)

13. Conn, A.R., Scheinberg, K., Vicente, L.N.: Introduction to Derivative-Free Optimization. Society for Industrial and Applied Mathematics, Philadelphia (2009)

14. Custódio, A.L., Dennis, J.E., Vicente, L.N.: Using simplex gradients of nonsmooth functions in direct search methods. IMA Journal of Numerical Analysis 28(4), 770-784 (2008)

15. Custódio, A.L., Rocha, H., Vicente, L.N.: Incorporating minimum Frobenius norm models in direct search. Computational Optimization and Applications 46(2), 265-278 (2010)

16. Custódio, A.L., Vicente, L.N.: Using sampling and simplex derivatives in pattern search methods. SIAM Journal on Optimization 18(2), 537-555 (2007)

17. Eberhart, R., Kennedy, J.: A new optimizer using particle swarm theory. In: MHS'95. Proceedings of the Sixth International Symposium on Micro Machine and Human Science, pp. 39-43. IEEE (1995)

18. Fermi, E., Metropolis, N.: Numerical solution of a minimum problem. Tech. rep., Los Alamos National Laboratory, Los Alamos (1952)

19. Frimannslund, L., Steihaug, T.: A generating set search method using curvature information. Computational Optimization and Applications 38(1), 105-121 (2007)

20. Gerstenhaber, M.: Theory of convex polyhedral cones. In: T.C. Koopmans (ed.) Activity Analysis of Production and Allocation, chap. 18, pp. 298-316. Wiley, New York (1951)

21. Gilmore, P., Kelley, C.T.: An implicit filtering algorithm for optimization of functions with many local minima. SIAM Journal on Optimization 5(2), 269-285 (1995)

22. Gratton, S., Royer, C.W., Vicente, L.N.: A second-order globally convergent direct-search method and its worst-case complexity. Optimization 65(6), 1105-1128 (2016)

23. Holland, J.H.: Adaptation in Natural and Artificial Systems. University of Michigan Press, Ann Arbor (1975)

24. Hooke, R., Jeeves, T.A.: "Direct search" solution of numerical and statistical problems. Journal of the ACM $\mathbf{8}(2), 212-229(1961)$

25. Kelley, C.T.: Detection and remediation of stagnation in the Nelder-Mead algorithm using a sufficient decrease condition. SIAM Journal on Optimization 10(1), 43-55 (1999)

26. Kelley, C.T.: Implicit filtering. Society for Industrial and Applied Mathematics, Philadelphia (2011)

27. Kolda, T.G., Lewis, R.M., Torczon, V.: Optimization by direct search: New perspectives on some classical and modern methods. SIAM Review 45(3), 385-482 (2003) 
28. Kolda, T.G., Lewis, R.M., Torczon, V.: Stationarity results for generating set search for linearly constrained optimization. SIAM Journal on Optimization 17(4), 943-968 (2006)

29. Larson, J., Menickelly, M., Wild, S.M.: Derivative-free optimization methods. Acta Numerica 28, 287-404 (2019)

30. Lewis, R.M., Shepherd, A., Torczon, V.: Implementing generating set search methods for linearly constrained minimization. SIAM Journal on Scientific Computing 29(6), 2507-2530 (2007)

31. Lewis, R.M., Torczon, V.: Pattern search algorithms for bound constrained minimization. SIAM Journal on Optimization 9(4), 1082-1099 (1999)

32. Lewis, R.M., Torczon, V.: Pattern search methods for linearly constrained minimization. SIAM Journal on Optimization 10(3), 917-941 (2000)

33. Lucidi, S., Sciandrone, M., Tseng, P.: Objective-derivative-free methods for constrained optimization. Mathematical Programming 92(1), 37-59 (2002)

34. Moré, J.J., Wild, S.M.: Benchmarking derivative-free optimization algorithms. SIAM Journal on Optimization 20(1), 172-191 (2009)

35. Nocedal, J., Wright, S.J.: Numerical optimization, 2nd edn. Springer, New York (2006)

36. Torczon, V.: On the convergence of the multidirectional search algorithm. SIAM Journal on Optimization $\mathbf{1}(1), 123-145(1991)$

37. Torczon, V.: On the convergence of pattern search algorithms. SIAM Journal on Optimization 7(1), 1-25 (1997)

38. Tseng, P.: Fortified-descent simplicial search method: A general approach. SIAM Journal on Optimization 10(1), 269-288 (1999)

39. Vicente, L.N., Custódio, A.L.: Analysis of direct searches for discontinuous functions. Mathematical Programming 133(1-2), 299-325 (2012) 